\title{
Electronic Transport in Alloys with Phase Separation (Composites)
}

\author{
Joachim Sonntag1, Bertrand Lenoir ${ }^{2}$, Pawel Ziolkowski ${ }^{3 *}$ \\ ${ }^{1}$ TE Connectivity Sensors Germany GmbH, Dortmund, Germany \\ ${ }^{2}$ Institut Jean Lamour, UMR 7198 CNRS-Université de Lorraine, 2 Allée André Guinier-Campus ARTEM, \\ Nancy Cedex, France \\ ${ }^{3}$ German Aerospace Center, Institute of Materials Research, Köln, Germany \\ Email: sonntag.joachim45@gmail.com, bertrand.lenoir@univ-lorraine.fr,`Pawel.Ziolkowski@dlr.de
}

How to cite this paper: Sonntag, J., Lenoir, B. and Ziolkowski, P. (2019) Electronic Transport in Alloys with Phase Separation (Composites). Open Journal of Composite Materials, 9, 21-56.

https://doi.org/10.4236/ojcm.2019.91002

Received: September 10, 2018

Accepted: January 25, 2019

Published: January 28, 2019

Copyright (c) 2019 by author(s) and Scientific Research Publishing Inc. This work is licensed under the Creative Commons Attribution International License (CC BY 4.0).

http://creativecommons.org/licenses/by/4.0/

(c) (i) Open Access

\begin{abstract}
A measure for the efficiency of a thermoelectric material is the figure of merit defined by $Z T=S^{2} T / \rho \kappa$, where $S, \rho$ and $\kappa$ are the electronic transport coefficients, Seebeck coefficient, electrical resistivity and thermal conductiviy, respectively. $T$ is the absolute temperature. Large values for $Z T$ have been realized in nanostructured materials such as superlattices, quantum dots, nanocomposites, and nanowires. In order to achieve further progress, (1) a fundamental understanding of the carrier transport in nanocomposites is necessary, and (2) effective experimental methods for designing, producing and measuring new material compositions with nanocomposite-structures are to be applied. During the last decades, a series of formulas has been derived for calculation of the electronic transport coefficients in composites and disordered alloys. Along the way, some puzzling phenomenons have been solved as why there are simple metals with positive thermopower? and what is the reason for the phenomenon of the "Giant Hall effect"? and what is the reason for the fact that amorphous composites can exist at all? In the present review article, (1), formulas will be presented for calculation of $\sigma=(1 / \rho), \kappa, S$, and $R$ in composites. $R$, the Hall coefficient, provides additional informations about the type of the dominant electronic carriers and their densities. It will be shown that these formulas can also be applied successfully for calculation of $S, \rho, \kappa$ and $R$ in nanocomposites if certain conditions are taken into account. Regarding point (2) we shall show that the combinatorial development of materials can provide unfeasible results if applied noncritically.
\end{abstract}

\section{Keywords}

Hall Effect, Giant Hall Effect, Seebeck Coefficient (Thermopower), 
Electron Density, Conductivity, Thermal Conductivity, Composites, Nanocomposites, Percolation Theory

\section{Introduction}

The performance of a thermoelectric material for cooling of power generation applications, or more generally, for energy conversion, are directly related to the dimensionless figure of merit defined by

$$
Z T=\frac{S^{2} T}{\rho \kappa},
$$

where $\rho=1 / \sigma . S, \kappa, \sigma$ and $\rho$ are the Seebeck (or thermopower) coefficient, thermal conductivity, electrical conductivity, and electrical resitivty, respectively. $T$ is the absolute temperature. The larger is $Z T$, the larger the thermoelectric performance of the material is. For many decades, $Z T \approx 1$ was a practical upper limit realized in real materials. A further increase of $Z T$ was limited by the fact that the transport coefficients occurring in Equation (1) are generally not independent from each other. In spite of these restrictions, during the last decade, progress was achieved by so-called nanostructured materials such as superlattices, quantum dots, nanocomposites, and nanowires.

In order to achieve further progress in the field of nanostructured materials with improved $Z T$, (1) a fundamental understanding of the carrier transport in these complex materials is necessary, and (2) effective experimental methods for designing, producing and measuring new material compositions with nanocomposite-structures are to be applied.

Regarding point (1), today it is generally accepted that application of the established classical theories for calculation of the electronic transport as the Boltzmann transport equation (BTE) can no longer be applied for the nanocomposite materials, because many of the characteristic lengths in the nanocomposite materials are smaller than the electron de Broglie wavelength (see, e.g., [1], Figure 6 therein). Instead, modelling transport in nanocomposites is generally assumed to require more powerful tools such as non-equilibrium Green's functions, which explicitly take account of electron wave effects [1].

Regarding point (2), the combinatorial development of materials seems to be a proper method for experimental studies. From this method (applied to a certain material system) a large range of different compositions can be realized concurrently on one large substrat, for instance by deposition of thin films by simultaneous co-sputtering from two or three targets on a large substrat. Subsequently the transport coefficients can be measured with high lateral resolution applying measuring equipment such as the Potential-Seebeck Microprobe, the 3-Omegamethod and the 4-point method for $S, \kappa$, and $\sigma$, respectively. The advantage of these methods is the fact that quick and precise measurements of the transport coefficients at identical positions over the complete substrate is possible. How- 
ever, there is also a disadvantage of this method. Because in a continuous sample with a lateral concentration gradient the electrochemical potential $\tilde{\mu}$ is the same in the whole sample, both the topological structure (atomic configuration) and electronic structure may be completelly different from those ones, if the samples with a certain composition are produced individually.

In the following we shall challenge these two points of view. We shall show that (1), the classical theories can, after all, be applied successfully for nanocomposites, particularly with respect to the electronic transport and its relation with the atomic structure and (2), the combinatorial development of materials can provide unfeasible results if applied noncritically.

On the way to this awareness, studies of amorphous alloys, as for instance $a-\mathrm{Cr}_{1-x} \mathrm{Si}_{x}$ alloys, have played an important role. Additional microstructure analyses of a series of amorphous transition-metal-alloys [2] [3] [4] [5] [6], have shown that, for large concentration ranges, they are real nanocomposites composed of different (amorphous) phases, where the phase grains are found to be of the order of $1-2 \mathrm{~nm}$ [5] [6]. In correspondence to these analytical results, the $a-\mathrm{Cr}_{1-x} \mathrm{Si}_{x}$ thin films can be described as nanocomposites consisting of the phases $a-\mathrm{Cr}_{1-x_{A}} \mathrm{Si}_{x_{A}}(\equiv$ phase $A)$ and $a-\mathrm{Cr}_{1-x_{B}} \mathrm{Si}_{x_{B}} \quad(\equiv$ phase $B)$ where $x_{A} \approx 0.25$ and $x_{B} \approx 1$.

In Sections 2.1-2.4 formulas for $\sigma, \kappa, S$ and $R$ (the Hall coefficient) in composites will be derived and, in Section 2.5, compared with other published formulas. In Section 2.6 percolation elements will be additionally introduced in the formulas for two-phase composites. In Section 3.1 the classical formulas for the transport coefficients of the phases will be summarized applicable to large phase grains, which in Section 3.2 are extended to nanocomposites. In Sections 4-6 it will be shown that under certain conditions a discontinuity in the concentration dependence of the thermopower can occur, that the classical thermopower formula is to be supplemented by an additional term to be complete and that a noncritical application of the method of the combinatorial development of materials can provide unfeasible results.

The puzzling phenomenons: why there are simple metals with positive thermopower? and what is the reason for the phenomenon of the "Giant Hall effect"? and what is the reason for the fact that amorphous composites can exist at all? have been considered in the Section 5 and Section 3.2.1.

\section{Electronic Transport in Composites}

Let us consider a two phase-composite consisting of the phases $i=A, B$ in a symmetrical fashion regarding the average geometric form of the phase grains and without preferred orientations. Let us assume that each phase can be characterized by a set of transport coefficients $\sigma_{i}, \kappa_{e, i}, S_{i}$ and $R_{i}$ for the phase $i$, which are the electrical conductivity, electronic contribution to the thermal conductivity, Seebeck coefficient and the Hall coefficient, respectively, in the phase $i$. The corresponding transport coefficients of the composite, $\sigma$, 
$\kappa_{e}, S$ and $R$ are to be calculated, if the $\sigma_{i}, \kappa_{e, i}, S_{i}$ and $R_{i}$ are known. The discussion will be restricted to small temperature gradients, small and constant electric and magnetic fields, $\mathbf{E}$ and $\mathbf{H}$, respectively.

\subsection{Electrical Conductivity}

Applying effective medium theory (EMT), let us derive the relation between $\sigma_{i}$ and $\sigma$, the electrical conductivities in the phases $i$ and the composite, respectively. The strategy underlying the EMT is the following: a single phase grain of the phase $i$ is considered to be completely embedded in an effective medium consisting of the two phases randomly arranged and characterized by the total transport coefficients. At the boundary face between this single phase grain and the surrounding effective medium continuity of the current densities and potentials and their gradients are to be saved.

The local electric current density $\mathrm{J}_{i}$ can be written as

$$
\mathrm{J}_{i}=\sigma_{i} \mathrm{E}_{i}=-\sigma_{i} \operatorname{grad} \varphi_{i} .
$$

In analogy to Equation (2) we write for the electric current density $\mathrm{J}$ in the specimen

$$
\mathrm{J}=\sigma \mathrm{E}=-\sigma \operatorname{grad} \varphi .
$$

$\varphi_{i}$ and $\varphi$ are the local and total electrostatic potential and $E_{i}=-\operatorname{grad} \varphi_{i}$ and $E=-\operatorname{grad} \varphi$ are the local and total electric field, respectively.

Now we demand that the total current density is equal to the average of the local current density,

$$
\mathrm{J}=\left\langle\mathrm{J}_{i}\right\rangle,
$$

and the same for the potential

$$
\operatorname{grad} \varphi=\left\langle\operatorname{grad} \varphi_{i}\right\rangle .
$$

Let us assume a spherical inclusion of the phase $i$ with the radius $r_{0}$, embedded in an uniform medium with the average conductivity $\sigma$ and that for this enclosed phase $i$ the local transport equation Equation (2) holds. With Equations (3)-(5) we get

$$
\mathrm{J}=-\sigma\left\langle\operatorname{grad} \varphi_{i}\right\rangle=-\left\langle\sigma_{i} \operatorname{grad} \varphi_{i}\right\rangle .
$$

The local potential $\varphi_{i}$ obeys the Laplace equation

$$
\Delta \varphi_{i}=0,
$$

where the boundary conditions

$$
\begin{aligned}
& \varphi=\varphi_{i}, \\
& \mathbf{J}^{n}=\mathbf{J}_{i}^{n}
\end{aligned}
$$

at $r=r_{0}$ are to be fulfilled, which is equivalent to

$$
\sigma \operatorname{grad}_{r} \varphi=\sigma_{i} \operatorname{grad}_{r} \varphi_{i}
$$

at $r=r_{0} \cdot \operatorname{grad}_{r}$ is the gradient into the radial direction. $\mathrm{J}_{i}^{n}$ and $\mathrm{J}^{n}$ are the normal components of the current density in the sphere $i$ and the surrounding 
effective medium, respectively. Equation (7) has the solution

$$
\varphi_{i}=\varphi_{0}+r a_{i} \cos \vartheta
$$

within the sphere $i$, and

$$
\varphi=\varphi_{0}+\left(b r+c r_{0}^{3} / r^{2}\right) \cos \vartheta
$$

within the effective medium. $\vartheta$ is the angle between the direction of $\mathbf{E}$ and the position vector $\mathbf{r}$ with $|\mathbf{r}|=r . a_{i}, b, c$ and $\varphi_{0}$ are constants. With

$$
\operatorname{grad}_{r} \varphi_{i}=a_{i} \cos \vartheta
$$

following from Equation (11), Equation (6) can be written as

$$
\sigma\left\langle a_{i}\right\rangle=\left\langle a_{i} \sigma_{i}\right\rangle .
$$

With the boundary condition Equation (8) it follows that

$$
a_{i}=b+c,
$$

and with Equations (10)-(12) for $r=r_{0}$,

$$
\sigma(b-2 c)=\sigma_{i} a_{i} .
$$

Equations (15), (16) resolved for $a_{i}$ providing

$$
a_{i}=\frac{3 b \sigma}{\sigma_{i}+2 \sigma}
$$

and introduced in Equation (14) provide

$$
\sigma\left\langle\frac{1}{\sigma_{i}+2 \sigma}\right\rangle=\left\langle\frac{\sigma_{i}}{\sigma_{i}+2 \sigma}\right\rangle .
$$

Replacing the averages $\langle\ldots\rangle$ by $\Sigma_{i} v_{i} \ldots$ we get

$$
\sum_{i} v_{i} \frac{\sigma_{i}-\sigma}{\sigma_{i}+2 \sigma}=0
$$

where $v_{i}$ is the volume fraction of the phase $i$.

Equation (19) is the EMT formula for $\sigma$. It holds for composites with more than two phases as well. The first authors who derived Equation (19) independently from each other, were Odelevskii [7] and Landauer [8].

\subsection{Thermal Conductivity}

The schema shown in Section 2.1 can be applied to all the other transport parameters provided that a current density can be defined which is a function of a gradient of a potential, analoguously to Equation (2). For the thermal conductivity the corresponding relations read

$$
\mathrm{J}_{\mathrm{Q}}=-\kappa_{e} \operatorname{grad} T
$$

and

$$
\mathrm{J}_{\mathrm{Q} i}=-\kappa_{e, i} \operatorname{grad} T_{i},
$$

where $\mathrm{J}_{\mathrm{Q}}$ and $\mathrm{J}_{\mathrm{Q} i}$ are the total and local electronic thermal current density, respectively. Applying the same formalism as described in Section 2.1, however 
where the potentials $\varphi$ and $\varphi_{i}$ are replaced by the potentials $T$ and $T_{i}$, respectively, it follows that

$$
\sum_{i} v_{i} \frac{\kappa_{e, i}-\kappa_{e}}{\kappa_{e, i}+2 \kappa_{e}}=0
$$

For the total thermal conductivity composed of both an electronic contribution $\kappa_{e}$ and an nonelectronic one $\kappa_{n e}$, it follows an analogous formula

$$
\sum_{i} v_{i} \frac{\kappa_{i}-\kappa}{\kappa_{i}+2 \kappa}=0
$$

where $\kappa_{i}$ is given by

$$
\kappa_{i}=\kappa_{e, i}+\kappa_{n e, i}
$$

provided that interactions between the various modes of heat transport can be neglected. $\kappa_{n e, i}$ is the non-electronic contribution to $\kappa_{i}$.

\subsection{Thermopower}

The following derivation will be based on $\mathrm{J}$ and $\mathrm{J}_{\mathrm{S}}$, the electric and entropy-flux density, respectively. The corresponding local flux densitities, $\mathrm{J}_{i}$ and $\mathrm{J}_{S, i}$, can be written as [9] [10]

$$
\begin{gathered}
\mathrm{J}_{i}=\sigma_{i}\left[\operatorname{grad}\left(\tilde{\mu}_{i} /|e|\right)-S_{i} \operatorname{grad} T\right] \\
\mathrm{J}_{S, i}=\sigma_{i} S_{i} \operatorname{grad}\left(\tilde{\mu}_{i} /|e|\right)-\left(\sigma_{i} S_{i}^{2}+\kappa_{e, i} / T\right) \operatorname{grad} T,
\end{gathered}
$$

where $|e|$ is the elementary charge. ( $-|e|$ is the charge of the electron.) $\tilde{\mu}_{i}$ is the electrochemical potential in the phase $i$.

According to the strategy underlying the EMT, we demand continuity of the entropy-flux density and the electrochemical potential and their gradients, at the boundary face between a single phase grain and its surrounding (effective medium), where additionally $J=J_{i}=0$ is to be fulfilled. Setting $J_{i}=0$ in Equation (25), and inserting into Equation (26), one obtains for the local entropy-flux density,

$$
\mathrm{J}_{S, i}=-\frac{\kappa_{e, i}}{S_{i} T} \operatorname{grad}\left(\tilde{\mu}_{i} /|e|\right) .
$$

In analogy to Equation (27) we write for the total entropy-flux density $J_{S}$ in the specimen

$$
\mathrm{J}_{S}=-\frac{\kappa_{e}}{S T} \operatorname{grad}(\tilde{\mu} /|e|),
$$

where $\tilde{\mu}$ is the electrochemical potential of the composite.

The Equations (27) and (28) have the same structure as Equations (2) and (3); that is why, we can apply the same formalism as described in Section 2.1, however with other starting equations,

$$
\begin{gathered}
\mathrm{J}_{S}=\left\langle\mathrm{J}_{S, i}\right\rangle, \\
\operatorname{grad} \tilde{\mu}=\left\langle\operatorname{grad} \tilde{\mu}_{i}\right\rangle,
\end{gathered}
$$


and we obtain finally

$$
\sum_{i} v_{i} \frac{\kappa_{e, i} / S_{i}-\kappa_{e} / S}{\kappa_{e, i} / S_{i}+2 \kappa_{e} / S}=0
$$

Considering the Wiedemann-Franz rule, Equation (31) can be transformed to

$$
\sum_{i} v_{i} \frac{\sigma_{i} / S_{i}-\sigma / S}{\sigma_{i} / S_{i}+2 \sigma / S} \approx 0
$$

Equation (32) was derived on basis $J \& J_{Q}$, Equation (31) on basis $J \& J_{S}$ (Sonntag, [11] and [12], respectively). For the one-band models, it makes little difference whether one chooses to interpret thermoelectric phenomenons on the basis of $\mathrm{J} \& \mathrm{~J}_{\mathrm{Q}}$ or $\mathrm{J} \& \mathrm{~J}_{\mathrm{S}}$. However, for two-band or multiband models characteristic for semiconductors, the use of $J \& J_{Q}$ leads to complications, which are not to be expected, if $\mathrm{J} \& \mathrm{~J}_{\mathrm{S}}$ is used as a basis (Harman and Honig [9], p. 28).

\subsection{Hall Coefficient}

Let us consider a non-magnetic two-phase composite. Under the same conditions as assumed in Section 2.1 the local electric current density in a single grain of the phase $i(i=A$ or $B)$ can be written as

$$
\mathrm{J}_{i}=\vec{\sigma}_{i} \mathbf{E}_{i},
$$

where $\mathbf{E}_{i}$ and $\vec{\sigma}_{i}$ are the electric field and the magnetoconductivity tensor [13] in this grain. For the electric current density outside of this grain we write analogously

$$
\mathbf{J}=\ddot{\sigma} \mathbf{E},
$$

where $\mathbf{E}$ and $\ddot{\sigma}$ are the electric field and the magnetoconductivity tensor outside of this grain (effective medium). For the determination of the coefficients in $\vec{\sigma}_{i}$ we start with the equation for $\mathrm{J}_{i}$ under the influence of an electric and magnetic field, [9] [14] [15]

$$
\mathrm{J}_{i}=e_{i}^{2} \mathrm{~K}_{11, i} \mathbf{E}_{i}+\frac{e_{i}^{3}}{m_{i}} \mathbf{K}_{12, i}\left(\mathbf{E}_{i} \times \mathbf{B}\right)+\frac{e_{i}^{4}}{m_{i}^{2}} \mathbf{K}_{13, i} \mathbf{B}\left(\mathbf{E}_{i} \cdot \mathbf{B}\right) .
$$

$\mathrm{K}_{r s, i}$ are the transport integrals defined by

$$
\mathrm{K}_{r s, i}=-\frac{4}{3 m_{i}} \int \frac{E^{r} \tau_{i}^{s}}{1+\mu_{i}^{2} \mathbf{B}^{2}} \frac{\partial f_{i}^{0}(E, T)}{\partial E} N_{i}(E) \mathrm{d} E
$$

with the Fermi-Dirac distribution function,

$$
f_{i}^{0}(E, T)=\frac{1}{1+\mathrm{e}^{\frac{E-\tilde{\mu}_{i}}{k_{B} T}}} .
$$

$N_{i}(E), m_{i}$ and $\tau_{i}$ are the density of states, the effective mass and the relaxation time, respectively, of the carriers in the phase i. $E$ and $k_{B}$ are the energy and the Boltzmann constant, respectively. $e_{i}=-|e|$ and $+|e|$ for electrons and holes, respectively. The third summand in Equation (35) disappears 
only if $\mathbf{E}_{i}$ (or $\mathbf{E}_{i}$ ) is always perpendicular to $\mathbf{B}$. In a composite, however, $\mathbf{B}$ and $\mathbf{E}_{i}$ (or $\mathbf{E}_{i}$ ), are generally not perpendicular to each other because of the spherical boundary between a phase grain and its surrounding. Without loss of generality, the external fields applied to the sample, $\mathbf{E}_{e x t}$ and $\mathbf{B}$, have the directions of the $X$ and $Z$ axes, respectively. Then Equation (33) and Equation (35) lead to

$$
\vec{\sigma}_{i}=\frac{\sigma_{i}}{1+\xi_{i}^{2}}\left(\begin{array}{ccc}
1 & \xi_{i} & 0 \\
-\xi_{i} & 1 & 0 \\
0 & 0 & 1+v_{i} \xi_{i}^{2}
\end{array}\right),
$$

where $\xi_{i} \equiv \mu_{i} B=\sigma_{i} R_{i} B$. Analogously we write for $\ddot{\sigma}$,

$$
\vec{\sigma}=\frac{\sigma}{1+\xi^{2}}\left(\begin{array}{ccc}
1 & \xi & 0 \\
-\xi & 1 & 0 \\
0 & 0 & 1+v \xi^{2}
\end{array}\right)
$$

with $\xi \equiv \mu B=\sigma R B . \quad v_{i}=1+\cos \alpha_{i}$ and $v=1+\cos \alpha$, where $\alpha_{i}$ and $\alpha$ are the angle between $\mathbf{E}_{i}$ and $\mathbf{B}$, respectively between $\mathbf{E}$ and $\mathbf{B} . \mu$ and $\mu_{i}$ are the average Hall mobility in the composite and the local Hall mobility in the phase $i$, respectively.

At the interface between a single phase grain and its surrounding continuity of the normal components of the current density and the tangential components of the potential gradient are to be fulfilled. For the limiting case $\mathbf{B}=0$, this demand is fulfilled by

$$
f\left(\sigma, \sigma_{i}\right) \equiv \sigma_{A} \sigma_{B}+\sigma \sigma_{A}\left(3 v_{A}-1\right)+\sigma \sigma_{B}\left(3 v_{B}-1\right)-2 \sigma^{2}=0
$$

following from the EMT formula for $\sigma$, Equation (19).

For the case $\mathbf{B} \neq 0$, the tensor properties of $\vec{\sigma}_{i}$ and $\vec{\sigma}$, Equation (38) and Equation (39), are to be taken into account. Equation (40) expressed in tensor form reads

$$
\vec{\sigma}_{A} \vec{\sigma}_{B}+\vec{\sigma} \vec{\sigma}_{A}\left(3 v_{A}-1\right)+\vec{\sigma} \vec{\sigma}_{B}\left(3 v_{B}-1\right)-2 \vec{\sigma} \vec{\sigma}=0
$$

where the identities $\vec{\sigma}_{A} \vec{\sigma}_{B}=\vec{\sigma}_{B} \vec{\sigma}_{A}$ and $\vec{\sigma} \vec{\sigma}_{i}=\vec{\sigma}_{i} \vec{\sigma}$ have been used. Equation (41) determines the coefficients of Equation (39) as a function of the coefficients of Equation (38). Inserting Equation (38) and Equation (39) into Equation (41) and comparing coefficients for the tensor elements, we get

$$
\xi=\frac{\sigma_{A} \sigma_{B}\left(\xi_{A}+\xi_{B}\right)+\sigma \sigma_{A} \xi_{A}\left(3 v_{A}-1\right)+\sigma \sigma_{B} \xi_{B}\left(3 v_{B}-1\right)}{4 \sigma^{2}-\sigma \sigma_{A}\left(3 v_{A}-1\right)-\sigma \sigma_{B}\left(3 v_{B}-1\right)},
$$

following from the tensor elements $\sigma_{x y}$ or $\sigma_{y x}$, where quadratic and higher powers of $\xi, \xi_{i}$ are neglected, i.e., Equation (42) and the following Equations (43), (44) are low-field approximations. Within this approximation the parameters $v_{i}$ and $v$ do not have an influence on the result. From the tensor elements $\sigma_{x x}, \sigma_{y y}$, or $\sigma_{z z}$, Equation (40) follows.

Substituting $\xi$ and $\xi_{i}$ in Equation (42) by $R$ and $R_{i}$ and considering Equation (40) we get the $R$ formula for two-phase composites: 


$$
R=\frac{\sigma_{A}^{2} R_{A}\left[\sigma_{B}+\sigma\left(3 v_{A}-1\right)\right]+\sigma_{B}^{2} R_{B}\left[\sigma_{A}+\sigma\left(3 v_{B}-1\right)\right]}{\sigma\left(\sigma_{A} \sigma_{B}+2 \sigma^{2}\right)}
$$

The same formalism can also be applied to composites with more than two phases leading to relatively complex formulas for $R$. A self-contained and more manageable description of these $R$ formulas is given by

$$
\left(R \sigma^{2} \frac{\partial}{\partial \sigma}+\sum_{i=A, B, \cdots} R_{i} \sigma_{i}^{2} \frac{\partial}{\partial \sigma_{i}}\right) f\left(\sigma, \sigma_{i}\right)=0
$$

with

$$
f\left(\sigma, \sigma_{i}\right)=\left(\prod_{i=A, B, \cdots}\left(\sigma_{i}+2 \sigma\right)\right)\left(\sum_{i=A, B, \cdots} v_{i} \frac{\sigma_{i}-\sigma}{\sigma_{i}+2 \sigma}\right) .
$$

Equations (43)-(45) were firstly published by Sonntag [16].

\subsection{Comparison with Other Transport Formulas for Composites}

\subsubsection{Thermopower}

As we are interested in a direct comparison between the different thermopower formulas for composites or heterogeneous materials, the transport coefficients in the phase, $\kappa_{i}, S_{i}$, and $\sigma_{i}$, are set to be constant, although this is not realized in real composites caused by the condition of a common electrochemical potential as well as because of the specific features at $v_{i}<1 / 3$ as discussed in Section 3.2.2. Additionally, $\kappa_{i}=\kappa_{e, i}$ is set.

The thermopower formula derived by Airapetiants ([17], Equation (12) therein), called $S_{A i r}$, is

$$
S_{A i r}=\frac{\sum_{i} S_{i} \sigma_{i} g_{i}}{1-2 \sigma \sum_{i} g_{i}}
$$

with

$$
g_{i}=\frac{3 v_{i} \kappa}{\left(2 \sigma+\sigma_{i}\right)\left(2 \kappa+\kappa_{i}\right)} .
$$

In Figure 1, $S_{A i r}$ vs $v_{B}$ is shown for a composite consisting of two phases, $A$ and $B$, with the coefficients, $S_{A}=-18.5 \mu \mathrm{V} / \mathrm{K}, S_{B}=-370 \mu \mathrm{V} / \mathrm{K}$, $\sigma_{A}=7450 \Omega^{-1} \cdot \mathrm{cm}^{-1}, \sigma_{B}=7 \Omega^{-1} \cdot \mathrm{cm}^{-1}, \kappa_{A}=0.022 \mathrm{~W} \cdot \mathrm{K}^{-1} \cdot \mathrm{cm}^{-1}$, $\kappa_{B}=0.070 \mathrm{~W} \cdot \mathrm{K}^{-1} \cdot \mathrm{cm}^{-1}$ which were applied by Airapetiants [17], already, where $\kappa$ and $\sigma$ are calculated by Equation (23) and Equation (19), respectively. For comparison the thermopower is calculated with our formula, Equation (31) and Equation (23) for $\kappa$ applying the same numbers for $\kappa_{i}$ and $S_{i} \quad\left[\sigma_{i}\right.$ does not occur in Equation (31)]. The result $S$ is drawn in Figure 1 as well.

$S_{A i r}\left(v_{B}\right)$, Equation (46), and our $S\left(v_{B}\right)$, Equation (31), agree very well in the concentration range $v_{B}<0.62$, however, with increasing $v_{B}$ beyond $v_{B}=0.62$ there is increasing difference between $S_{A i r}\left(v_{B}\right)$ and $S\left(v_{B}\right)$. 


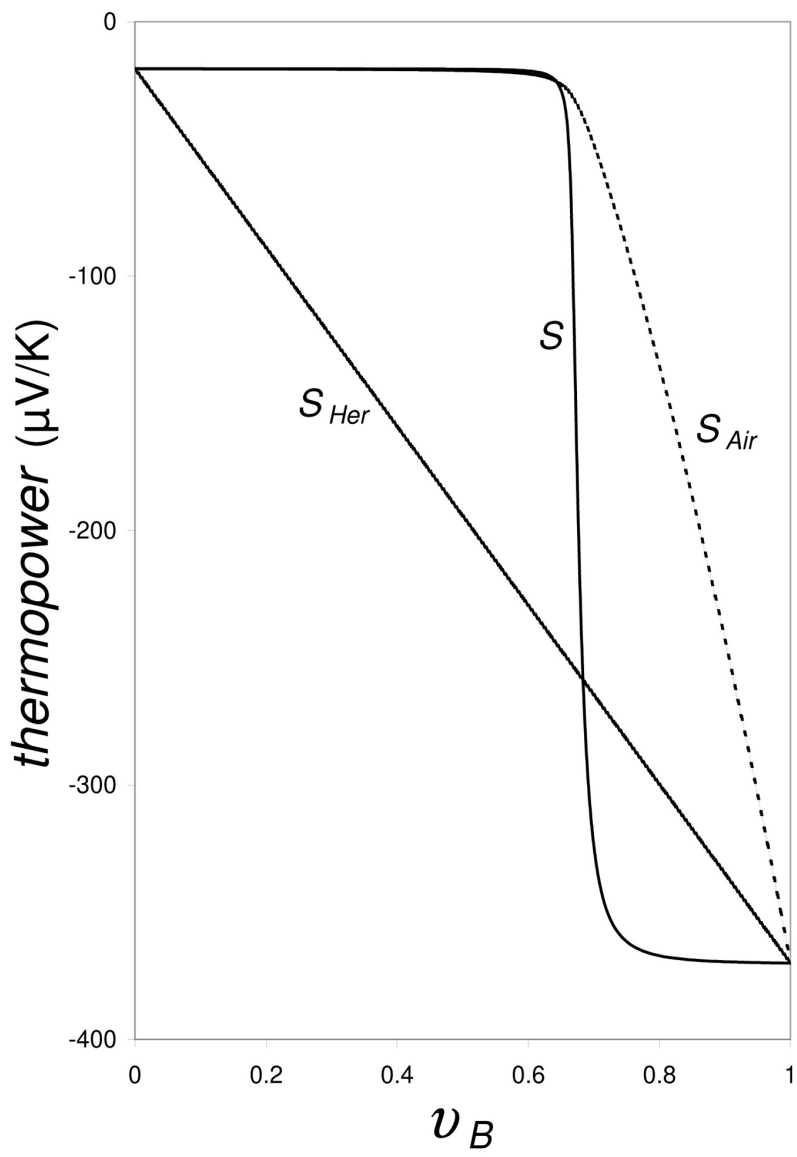

Figure 1. Comparison of $S_{A i r}, S_{H e r}$, and $S$ vs. $v_{B}$ calculated by Equation (46), Equation (56), and Equation (31), respectively, where $S_{A}=-18.5 \mu \mathrm{V} / \mathrm{K}, \sigma_{A}=7450 \Omega^{-1} \cdot \mathrm{cm}^{-1}$, $\kappa_{A}=0.022 \mathrm{~W} \cdot \mathrm{K}^{-1} \cdot \mathrm{cm}^{-1}, S_{B}=-370 \mu \mathrm{V} / \mathrm{K}$, $\sigma_{B}=7 \Omega^{-1} \cdot \mathrm{cm}^{-1}, \kappa_{B}=0.070 \mathrm{~W} \cdot \mathrm{K}^{-1} \cdot \mathrm{cm}^{-1} \quad$ [the numbers for $S_{i}, \sigma_{i}$, and $\kappa_{i}$ are taken from [17] (Figure 2 therein); the quotients of them, $S_{B} / S_{A}, \sigma_{B} / \sigma_{A}$, and $\kappa_{B} / \kappa_{A}$ correspond nearly to those applied in [webman] (Figure 2 therein).

The thermopower formula derived by Webman et al. ([webman], Equations (2.16), (2.17) therein),

$$
S_{W e b}=\frac{6 \kappa \sum_{i} v_{i} S_{i} D_{i}}{1-3 \sum_{i} v_{i} \kappa_{i} D_{i}}
$$

with $^{1}$

$$
D_{i}=\frac{\sigma_{i}}{\left(\kappa_{i}+2 \kappa\right)\left(\sigma_{i}+2 \sigma\right)},
$$

provides the same concentration dependence as those of Airapetiants [17], $S_{A i r}\left(v_{B}\right)$, shown in Figure 1, if $\kappa$ and $\sigma$ in Equations (48), (49) are again ${ }^{1}$ We have replaced the averages $\langle\ldots\rangle$ in the original equations by Webman et al. [webman] and Herring [herring] by $\sum_{i} v_{i} \ldots$, Equations (48) and (55), respectively. 
calculated by Equation (23) and Equation (19), respectively. This result seems to be surprizing, because at the end of the derivation of theirs formulas Webman et al. have written: "Our effective-medium result... differs from that previously derived by Airapetiants [17], as the latter treatment did not involve a completely self-consistent averaging procedure."

Also the thermopower formula derived by Halpern [19] (Equation (10) therein),

$$
S_{\text {Halp }}=\frac{S_{A} \sigma_{A} \kappa_{B}-S_{B} \sigma_{B} \kappa_{A}}{\sigma_{A} \kappa_{B}-\sigma_{B} \kappa_{A}}+\frac{\sigma_{A} \sigma_{B} \kappa\left(S_{B}-S_{A}\right)}{\sigma\left(\sigma_{A} \kappa_{B}-\sigma_{B} \kappa_{A}\right)},
$$

provides the same concentration dependence as those by Airapetiants [17], $S_{A i r}$; the same refers also to the thermopower formula derived by Balagurov ([20], Equation (7) therein),

$$
S_{B a l}=S_{A}+\frac{\sigma_{A} \sigma_{B}\left(S_{A}-S_{B}\right)}{\sigma_{A} \kappa_{B}-\sigma_{B} \kappa_{A}}\left(\frac{\kappa_{A}}{\sigma_{A}}-\frac{\kappa}{\sigma}\right)
$$

as well as to those by Bergman and Levy ([21], Equation (50) and Equation (9) therein),

$$
S_{B e r g}=S_{B}+\left(S_{A}-S_{B}\right) \frac{\gamma / \sigma-\gamma_{B} / \sigma_{B}}{\gamma_{A} / \sigma_{A}-\gamma_{B} / \sigma_{B}}
$$

with

$$
\begin{gathered}
\gamma=\kappa+T \sigma S^{2}, \\
\gamma_{i}=\kappa_{i}+T \sigma_{i} S_{i}^{2}
\end{gathered}
$$

if $\kappa$ and $\sigma$ in Equations (50)-(53) are again calculated by Equation (23) and Equation (19), and $\gamma_{i}$ and $\gamma$ in Equation (52) are replaced by $\kappa_{i}$ and $\kappa$, respectively, i.e., the second term in Equation (53) and Equation (54) is neglected [22] [23] (quantity of higher order).

Additionally, in Figure 1 it is drawn the concentration dependence of $S_{\mathrm{Her}}$ calculated by the formula by Herring ([24], second equation of Equation (38) therein]),

$$
S_{H e r}=\langle S\rangle-\left\langle(S-\langle S\rangle)\left(\frac{1}{3} \frac{\kappa}{\langle\kappa\rangle}+\frac{2}{3} \frac{\rho}{\langle\rho\rangle}\right)\right\rangle
$$

with $\rho=\sigma^{-1}$, derived for a randomly inhomogeneous medium. If $\langle S\rangle,\langle\kappa\rangle$ and $\langle\rho\rangle$ are interpreted as $\sum_{i} v_{i} S_{i}, \sum_{i} v_{i} \kappa_{i}$ and $\sum_{i} v_{i} \rho_{i}$, respectively, then Equation (55) leads to

$$
S_{\text {Her }}=\sum_{i} v_{i} S_{i}-\sum_{j} v_{j}\left(S_{j}-\sum_{i} v_{i} S_{i}\right)\left(\frac{1}{3} \frac{\kappa}{\sum_{i} v_{i} \kappa_{i}}+\frac{2}{3} \frac{\rho}{\sum_{i} v_{i} \rho_{i}}\right)
$$

with $\rho_{i}=\sigma_{i}^{-1}$. It is noteworthy that Bergman and Levy [21] have concluded that for a two-component isotropic composite their result, Equation (52), agree in first order of $\sigma_{A}-\sigma_{B}$ and $\gamma_{A}-\gamma_{B}$ with that by Herring [24], Equation (55), but disagree in the next order. 
Fishchuk [25] received for the conducting range of a two-component system in the form of a semiconductor with random insulating inclusions [25], $S=S_{A}$, in correspondence with $S_{A i r}$ and $S$ in Figure 1.

Summarizing, for the example considered in Figure 1, the surprising result follows: Although the formulas for $S_{A i r}, S_{W e b}, S_{H a l p}, S_{B a l}$, and $S_{B e r g}$ look very differently, all provide the same concentration dependence of the thermopower in the complete concentration range $0 \leq v_{B} \leq 1$, whereas there are considerable differences to both $S_{H e r}\left(v_{B}\right)$ and $S\left(v_{B}\right)$, Equation (31).

Which are the reasons for the differences between the thermopower formulas considered? All the thermopower formulas cited contain $S_{i}, \kappa_{i}$ and $\sigma_{i}$, whereas our Equation (31) contains only $S_{i}$ and $\kappa_{e, i}$ (or $S_{i}$ and $\sigma_{i}$, Wiedemann-Franz rule is used, Equation (32)). Also the thermopower formula derived by Xia and Zeng [26] for polycrystals, contains $S_{i}, \kappa_{i}$ and $\sigma_{i}$.

The reason for the differences is the neglection of the quadratic term of the thermopower-coefficient in the heat current density ("quantity of higher order") before $\mathrm{J}_{i}=0$ is set. Neglection of this term, " $\sigma_{i} S_{i}^{2}$ ", leads to an additional term in the resulting $\mathrm{J}_{S, i}$, Equation (27), after setting $\mathrm{J}_{i}=0$; this additional term depends on $\sigma$ as well. Such a term does not occur, if all the terms are maintained, before $\mathrm{J}_{i}=0$ is set. This statement is independent of the question whether the EMT formula is derived on the basis of $\mathrm{J} \& \mathrm{~J}_{\mathrm{Q}}$ or $\mathrm{J} \& \mathrm{~J}_{\mathrm{S}}$.

The starting equations applied by Webman et al. [18] (Equations (2.2b) and (2.2a) therein) read

$$
\begin{gathered}
\mathrm{J}=-\sigma \operatorname{grad} \varphi+P \operatorname{grad} T, \\
\mathrm{~J}_{Q}=-\kappa \operatorname{grad} T+P T \operatorname{grad} \varphi,
\end{gathered}
$$

where $P=S \sigma$. Setting $\mathrm{J}=0$, it follows that

$$
\operatorname{grad} T=\frac{\operatorname{grad} \varphi}{S},
$$

and replacing $\operatorname{grad} T$ in Equation (58) by Equation (59) we get

$$
\mathrm{J}_{Q}=\left(-\frac{\kappa}{S}+\sigma S T\right) \operatorname{grad} \varphi,
$$

Equation (60) contains $\sigma, \kappa$ and $S$. This does not occur if the complete formula,

$$
\mathrm{J}_{Q}=-\left(\kappa+S^{2} \sigma T\right) \operatorname{grad} T+P T \operatorname{grad} \varphi
$$

is applied as the starting formula instead of Equation (58). In Equation (28) only $\kappa_{e}$ and $S$ occur.

Also the other authors have applied analogous formulas: Bergman and Levy ([21], Equation (50) and Equation (9) therein); Xia and Zeng [26] (Equation (5) therein). Halpern has also neglected the quadratic term in $S$ (first line in [19] on page L219), although he comes to the reasonable result that for (two-phase) metal-insulator composites, $S \approx S_{A}$, where $S_{A}$ is the thermopower of the metallic phase. This result agrees with the result of Sonntag for metal-insulator 
composites ([11], Equation (59) therein).

The basis of all the derivations done in [11] [18] [19] [21] [26] was J \& $\mathrm{J}_{\mathrm{Q}}$. As pointed out by Harman and Honig ([9], p. 28), for desribing thermoelectric phenomenons, $\mathrm{J}_{\mathrm{S}}$ is more universal as $\mathrm{J}_{\mathrm{Q}}$. That is why, in [12] $\mathrm{J} \& \mathrm{~J}_{\mathrm{S}}$ has been applied as a basis. There are differences between Equation (31) derived in [12] on basis J \& $\mathrm{J}_{\mathrm{S}}$ and the formula derived on basis $\mathrm{J}_{\mathrm{C}} \& \mathrm{~J}_{\mathrm{Q}}$ (Equation (30) in [11]). While in the last one the chemical potentials of the phases, $\tilde{\mu}_{i}^{0}$, occur, in the former one only the common electrochemical potential, $\tilde{\mu}$, occurs mediated through the $S_{i}$ described by Equation (73).

\subsubsection{Hall Coefficient}

The EMT formula for the Hall coefficient of two-phase composites derived by Cohen and Jortner [27] is

$$
\sum_{i=A, B} v_{i} \frac{\sigma_{i}^{2} R_{i}-\sigma^{2} R}{\left(\sigma_{i}+2 \sigma\right)^{2}}=0 .
$$

As will be argued in the following, Equation (62) seems to be a good approximation for two-phase composites if $\sigma_{A} \approx \sigma_{B}$, but not if $\sigma_{A} \gg \sigma_{B}$, as typical for metal-insulator composites.

For three examples of two-phase composites, in Figure 2(b), Figure 2(d), and Figure 2(f), the concentration dependence of $R$ related to its value at $v_{A}=1$ is shown, calculated by Equation (43), and compared with Equation (62), denoted as "C\&J". In Figure 2(a), Figure 2(c), and Figure 2(e), the corresponding concentration dependence of the Hall mobility $\mu(=\sigma R)$ related to its value at $v_{A}=1$ is shown, where $\sigma$ is calculated by Equation (19). There are two essential differences between the the two solutions Equation (62) and Equation (43):

(1) The most striking difference appears in Figure 2(a) and Figure 2(c): The "C\&J" curves decrease dramatically with increasing $v_{A}$ and pass through a pronounced minimum at $v_{A}=1 / 3$, although $\mu_{A}=\mu_{B}$ and $\mu_{A}>\mu_{B}$, respectively. In contrast, the $\mu$ curves calculated by Equation (43) agree with the expectation: Figure 2(a): $\mu$ agrees with $\mu_{A}$ for all $v_{A}$; Figure 2(c) and Figure 2(e): $\mu$ increases and decreases with increasing $v_{A}$, respectively.

A possible interpretation for such dramatic decrease of $\mu$ at $v_{A}=1 / 3$ ("C\&J" curves) could be additional scattering centres in the added phase boundaries. Such an effect by the phase boundaries is expected to be the more pronounced the smaller the sizes of the phase grains, $D_{i}$. However, the C\&J formula [27] [28] does not contain $D_{i}$.

The differences between Equation (43) and the curves "C\&J" increase with increasing difference between $\sigma_{A}$ and $\sigma_{B}$. On the other hand, for the limiting case, $\sigma_{A}=\sigma_{B}$, Equation (62) and Equation (43) agree.

(2) Another striking difference between Equation (43) and Equation (62) is represented by the boundary case " $\sigma_{B}=0$ and $\sigma_{A} \neq 0$ ”, for which one obtains 

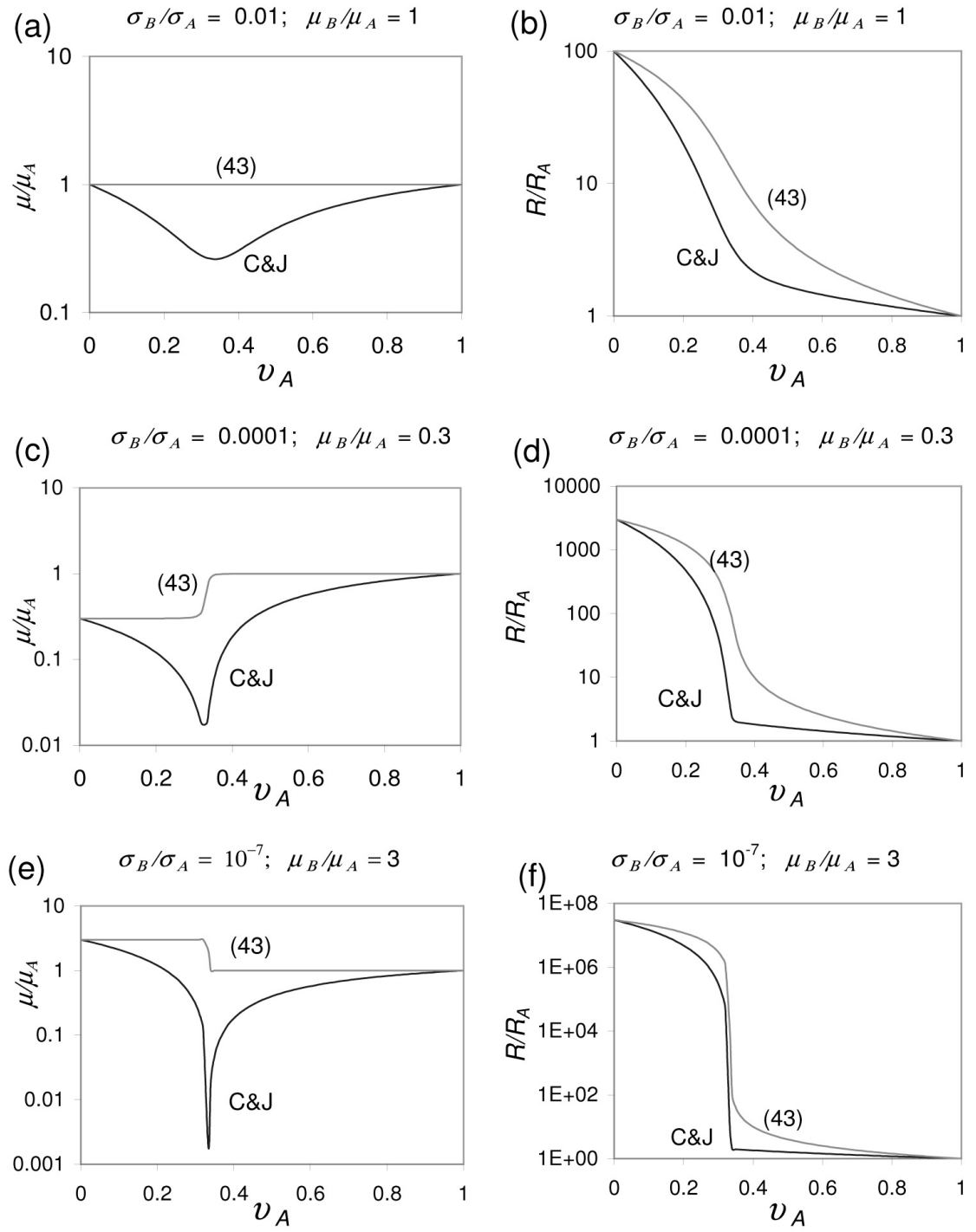

Figure 2. $\mu / \mu_{A}$ and $R / R_{A}$ versus $v_{A}$ calculated by Equation (62) ("C\&J") and Equation (43), where $\mu / \mu_{i}=R \sigma / R_{i} \sigma_{i}$. The "C\&J"-curves in (a) and (c) agree with [27] (Figure 1(b), Figure 1(c) therein) and [28] (Figures.13,14 therein), where the same examples are chosen.

$$
\frac{1}{R^{(43)}}=\frac{1}{R_{A}} \frac{\left(3 v_{A}-1\right)}{2}
$$

and

$$
\frac{1}{R^{\mathrm{C \& J}}}=\frac{1}{R_{A}} \frac{\left(3 v_{A}+1\right)}{4},
$$

respectively, and for $\sigma$, Equation (19) gives

$$
\sigma=\sigma_{A} \frac{\left(3 v_{A}-1\right)}{2} \text {. }
$$

Starting at $v_{A}=1$, with decreasing $v_{A}$ both $\sigma$ and $\frac{1}{R^{(43)}}$ decrease con- 
tinuously until they vanish at $v_{A}=1 / 3$. This result corresponds to the fact that for $v_{A}<1 / 3$ there is no longer a connected metal cluster through the composite (in correspondence with the assumption made earlier that the phase grains are spherical without preferred orientations and arranged in a symmetrical fashion). This result is, however, not reflected by Equation (64) which gives $\frac{1}{R^{\text {C\&J }}}>0$ even for $v_{A}<1 / 3$, where all the metallic granules are separated by adjacent insulating phase regions, that is, electron transport through the sample does not happen, if additional tunneling is excluded.

These two differences, (1) and (2), suggest the fact that Equation (63) represents the physical situation better than Equation (64).

\subsection{Composites with Non-Spherical Phase Grains}

An essential assumption for the derivation of the EMT formulas, Equations (19), (22), (31), (32), (43) and (44) was the fact that the phase grains are spherical. In real composites this assumption is often not fulfilled, especially not for manmade composites with large phase grains. A typical feature for these composites is the fact that the transition from an infinite phase $i$ cluster through the composite does not occur at $v_{i}=1 / 3$ but at larger values called the percolation edge. For such cases McLachlan and co-workers [30] [31] have modifyed the EMT formulas by introduction of percolation elements in the formulas for two-phase composites leading to the generalized form (GEMT)

$$
v_{A} \frac{\sigma_{A}^{1 / t}-\sigma^{1 / t}}{\sigma_{A}^{1 / t}+A \cdot \sigma^{1 / t}}+v_{B} \frac{\sigma_{B}^{1 / t}-\sigma^{1 / t}}{\sigma_{B}^{1 / t}+A \cdot \sigma^{1 / t}}=0
$$

and

$$
v_{A} \frac{\kappa_{A}^{1 / t}-\kappa^{1 / t}}{\kappa_{A}^{1 / t}+A \cdot \kappa^{1 / t}}+v_{B} \frac{\kappa_{B}^{1 / t}-\kappa^{1 / t}}{\kappa_{B}^{1 / t}+A \cdot \kappa^{1 / t}}=0
$$

where $A$ is given by $A=\left(1-v_{c}\right) / v_{c} \cdot v_{c}$ is the volume fraction of the phase $A$, where the actual percolation threshold is assumed to occur. $t$ represents the asymmetry of the microstructure. Vaney et al. [29] have applied this idea to the EMT formula for the thermopower. With this modification Equation (32) reads now

$$
v_{A} \frac{\left(\sigma_{A} / S_{A}\right)^{1 / t}-(\sigma / S)^{1 / t}}{\left(\sigma_{A} / S_{A}\right)^{1 / t}+A \cdot(\sigma / S)^{1 / t}}+v_{B} \frac{\left(\sigma_{B} / S_{B}\right)^{1 / t}-(\sigma / S)^{1 / t}}{\left(\sigma_{B} / S_{B}\right)^{1 / t}+A \cdot(\sigma / S)^{1 / t}} \approx 0,
$$

Vaney et al. [29] have studied real composites composed of a crystalline phase " $\mathrm{Bi}_{0.4} \mathrm{Sb}_{1.6} \mathrm{Te}_{3}$ " (三phase $A$ ) and a glassy phase " $\mathrm{Si}_{10} \mathrm{As}_{15} \mathrm{Te}_{75}$ " (三phase $B$ ) produced by spark plasma sintering (SPS) of powder mixtures with different volume fractions $v_{A}=0.1, \cdots, 0.5$.

Inserting the $\sigma_{i}$ and $S_{i}$ data (given by Vaney et al. [29]) in Equation (32), it follows the concentration dependence of $S$ on $v_{A}$ as drawn in Figure 3(a). For $v_{A}>1 / 3$ the calculated $S$ on $v_{A}$ curve agree with the experimental data. For $v_{A}<1 / 3$ the EMT formulas are no longer a good description, because the 


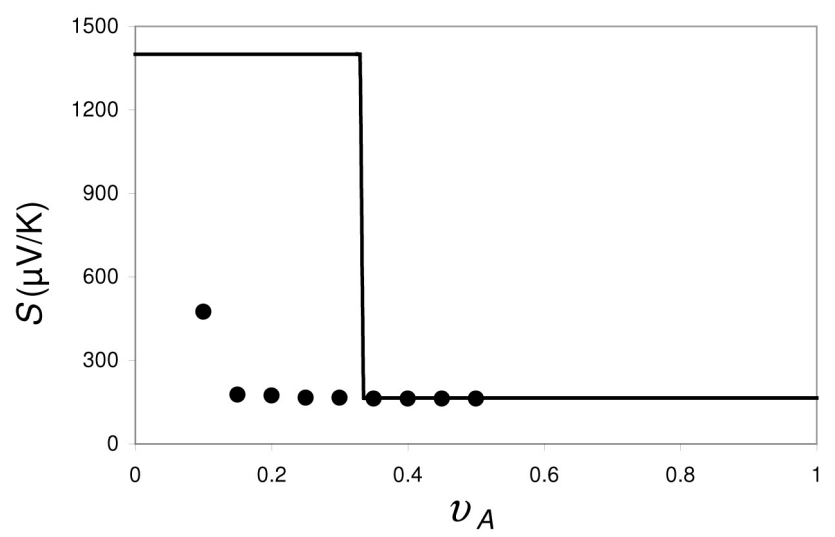

(a)

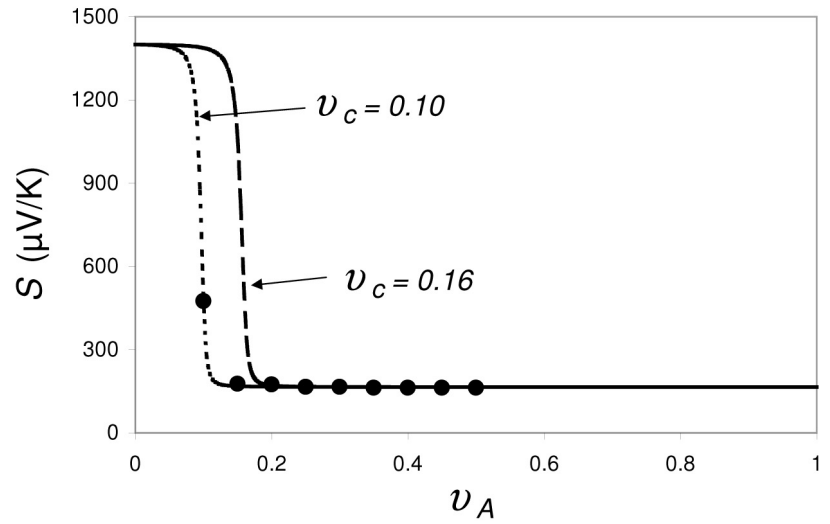

(b)

Figure 3. $S$ versus $v_{A}$ for $\left[\mathrm{Bi}_{0.4} \mathrm{Sb}_{1.6} \mathrm{Te}_{3}\right]_{v_{A}}\left[\mathrm{Si}_{10} \mathrm{As}_{15} \mathrm{Te}_{75}\right]_{1-v_{A}}$ composites produced by spark plasma sintering (SPS) [29] and comparison with the experimental data (full points, $T=300 \mathrm{~K}$ ). (a), calculated by Equation (32), (b), calculated by the GEMT equation, Equation (68), for $v_{c}=0.16$ and $v_{c}=0.10 ; t=2$ for both curves. For the calculations the same values have been applied as given by Vaney et al. [29]: $\sigma_{1}=0.040 \Omega^{-1} \cdot \mathrm{m}^{-1}$ and $S_{A}=165 \mu \mathrm{V} / \mathrm{K}$ for the crystalline phase $\left(\mathrm{Bi}_{0.4} \mathrm{Sb}_{1.6} \mathrm{Te}_{3}\right)$, $\sigma_{2}=2.95^{-9} \Omega^{-1} \cdot \mathrm{m}^{-1}$ and $S_{B}=1400 \mu \mathrm{V} / \mathrm{K}$ for the glassy phase $\left(\mathrm{Si}_{10} \mathrm{As}_{15} \mathrm{Te}_{75}\right)$.

phase grains in the man-made composites as studied in [29] are arranged randomly (accidentally), that is, the transition from a continuous $A$ phase cluster through the composite to separated $A$ phase grains does not occur at $v_{A}=1 / 3$, but it is shifted to smaller values of $v_{A}$. These percolation effects are considered by the generalized form (GEMT), Equation (68).

In Figure 3(b), $S$ versus $v_{A}$ is drawn calculated by Equation (68) for $t=2$ and $v_{c}=0.16$ (as applied in Figure 7(a) of [29]) as well as for $t=2$ and $v_{c}=0.10$. As can be seen in Figure 3(b), for the parameters $t=2$ and $v_{c}=0.10$ there is an excellent agreement with the experimental data.

Both in [29] and in Figure 3, the values for $S_{i}$ and $\sigma_{i}$ were assumed to be independent of $v_{i}$. This is, however, only an approximation, because the 
electrochemical potential, $\tilde{\mu}$, in the two phases is generally different, as long as the phases are separated from each other. In the composite, however, $\tilde{\mu}$ must be uniform. This is ensured by an electron transfer $\delta n$ between the phases. The temperature dependent part of $\delta n$ leads to a change of $\mathrm{d} \tilde{\mu} / \mathrm{d} T$. This provides an additional contribution to the thermopower, $\Delta S=\frac{1}{|e|} \frac{\mathrm{d} \tilde{\mu}}{\mathrm{d} T}$ as will be discussed in Section 3.1. For a calculation of $\mathrm{d} \tilde{\mu} / \mathrm{d} T$, knowledge of the band structure data of the phases is necessary. For the composite considered by Vaney et al. [29], the contribution of $\mathrm{d} \tilde{\mu} / \mathrm{d} T$ to $S$ is surely small, because $S_{A}$ and $S_{B}$ have the same sign. However, for composites with different signs of $S_{A}$ and $S_{B}$, the effect of $\mathrm{d} \tilde{\mu} / \mathrm{d} T$ can be essentially larger. That is, a really sensitive hardness test of Equation (32), respectively Equation (68), would be a composite with $S_{A}>0$ and $S_{B}<0$ or vice versa.

\section{Formulas for the Transport Coefficients of the Phases}

\subsection{Composites with Large Phase Grains}

"Large phase grains" means that the classical transport theory can be applied separately to each of the phase grains. This fact is immediarely plausible for crystalline phases, but also for amorphous phases as argued in [11] [32] [33]. First, we focus our attention on $\sigma, \kappa_{e}$ and $S$. In the framework of the kinetic transport equations, for a zero-magnetic field situation the transport coefficients of the phase $i$ can be written as [9] [10] [14] [15] [34] [35] [36] [37]

$$
\begin{gathered}
\sigma_{i}=e_{i}^{2} \mathrm{~K}_{11, i}, \\
\kappa_{e, i}=\frac{\mathrm{K}_{31, i}-\mathrm{K}_{21, i}^{2} / \mathrm{K}_{11, i}}{T}, \\
S_{i}^{0}=\frac{\mathrm{K}_{21, i} / \mathrm{K}_{11, i}-\tilde{\mu}_{i}^{0}}{e_{i} T} .
\end{gathered}
$$

where $\tilde{\mu}_{i}^{0}$ is the chemical potential for the phase $i$.

$S_{i}^{0}$ is the Seebeck coefficient of the phase $i$ for the (hypothetic) case that electron transfer does not happen between the different phases and that the band edge does not depend on $T$. Therefore an additional term, $\Delta S$, is to be introduced considering these effects realizing a common electrochemical potential in the composite,

$$
\tilde{\mu}=\tilde{\mu}_{i} .
$$

In the composite the Seebeck coefficient of the phase $i$ is given by [12]

$$
S_{i}=S_{i}^{0}+\Delta S=S_{i}^{0}+\frac{1}{|e|} \frac{\mathrm{d} \tilde{\mu}}{\mathrm{d} T},
$$

where $S_{i}^{0}$ is a scattering term. $\frac{1}{|e|} \frac{\mathrm{d} \tilde{\mu}}{\mathrm{d} T}$ is an additional term taking into account the change of $\tilde{\mu}$ with temperature. One consequence of Equation (73) is the fact that the thermopowers of the phases, $S_{i}$, dependent on $v_{i}$. For a 
calculation of $\mathrm{d} \tilde{\mu} / \mathrm{d} T$, knowledge of the band structure data of the phases is necessary. Assuming a two-phase composite consisting of the phase $A$ with electron conductivity and the phase $B$ with hole conductivity, $\mathrm{d} \tilde{\mu} / \mathrm{d} T$ can be calculated by ([38], Section 3.3. therein)

$$
\frac{\mathrm{d} \tilde{\mu}}{\mathrm{d} T}=\frac{\partial E_{C, A}}{\partial T}+\frac{\partial \tilde{\mu}_{A}^{0}}{\partial T}-\frac{\frac{\partial \tilde{\mu}_{A}^{0}}{\partial T}+\frac{\partial \tilde{\mu}_{B}^{0}}{\partial T}+\frac{\partial E_{C, A}}{\partial T}-\frac{\partial E_{V, B}}{\partial T}}{1+\frac{v_{A}\left(\frac{\partial \tilde{\mu}_{B}^{0}}{\partial p}-|e| \frac{\partial \varphi_{B}}{\partial n_{B}}\right)}{v_{B}\left(\frac{\partial \tilde{\mu}_{A}^{0}}{\partial n}-|e| \frac{\partial \varphi_{A}}{\partial n}\right)}},
$$

where $\partial E_{C, A} / \partial T$ and $\partial E_{V, B} / \partial T$ are the band edge shifts with temperature. $n$ $\left(\equiv n_{A}\right)$ is the electron density in the phase $A \cdot p\left(\equiv n_{B}\right)$ is the hole density in the phase $B . E_{C, A}$ and $E_{V, B}$ are the energies of the band edges of the conduction band (CB) and valence band (VB) in the phase $A$ and phase $B$, respectively. In Equation (74), $\partial \varphi_{i} / \partial T=0, \partial E_{C, A} / \partial n=0$, and $\partial E_{V, B} / \partial p=0$ is assumed. The first assumption corresponds to the fact that the electrostatic potential does not depend on $T$, the second and third ones are equivalent to the assumption that $E_{C, A}$ and $E_{V, B}$ do not depend on occupation of the CB and VB. From Equation (73) and Equation (74) it follows immediately the fact that $S_{i}$ depends on $v_{i}$.

Now, $\mathrm{d} \tilde{\mu} / \mathrm{d} T$ can be calculated by Equation (74) if

$$
\begin{gathered}
\frac{\partial \tilde{\mu}_{i}^{0}}{\partial T}=-\frac{\pi^{2} k_{B}^{2} T}{6 E_{F, i}}, \\
\frac{\partial \tilde{\mu}_{i}^{0}}{\partial n_{i}}=\frac{2 E_{F, i}}{3 n_{i}},
\end{gathered}
$$

are taken into account and if the band edge shifts for the $\mathrm{CB}$ and $\mathrm{VB}, \partial E_{C, A} / \partial T$ and $\partial E_{V, B} / \partial T$, are known. Equations (75) and (76) follow from the FermiDirac-statistics, where $\tilde{\mu}_{i}^{0}$ is given by

$$
\tilde{\mu}_{i}^{0}=E_{F, i}-\frac{\pi^{2} k_{B}^{2} T^{2}}{6} \frac{\mathrm{d}}{\mathrm{d} E}\left[\ln N_{i}(E)\right]_{E=E_{F, i}}=E_{F, i}-\frac{\pi^{2} k_{B}^{2} T^{2}}{12 E_{F, i}}
$$

(lowest order in the powers of $k_{B} T / E_{F, i}$ ) with

$$
E_{F, i}=\frac{h^{2}}{8 m_{i}}\left(\frac{3}{\pi}\right)^{2 / 3} n_{i}^{2 / 3},
$$

where $E_{F, i}$ is the Fermi energy and $m_{i}$ the effective mass in the phase $i$, respectively. $h$ is Planck's constant. The second equation in Equation (77) corresponds to the NFE-approximation.

For metallic phases, Equations (69)-(71) provides (NFE-approximation, [9] [10] [15])

$$
\begin{aligned}
\sigma_{i} & =2\left(\frac{\pi}{3}\right)^{1 / 3} \frac{\mathrm{e}^{2}}{h} L_{i} n_{i}^{2 / 3}, \\
\kappa_{e, i} & =\frac{16 \pi^{3}}{9} \frac{m_{i} L_{i} E_{F, i}}{h^{3}} k_{B}^{2} T,
\end{aligned}
$$




$$
S_{i}^{0}=\frac{\pi^{2} k_{B}^{2} T\left(1+r_{i}\right)}{3 e_{i} E_{F, i}},
$$

$r_{i}$ characterizes the scattering mechanism and represents the energy dependence of the mean free path $L_{i}$ in the phase $i$, according to $L_{i} \propto E^{r_{i}}$.

For composites with semiconducting phases, the $\sigma_{i}, \kappa_{e, i}$ and $S_{i}^{0}$ are to be calculated according to the rules for semiconducting solids, in correspondence with the two-band (or multiband) model (see, e.g., Harman and Honig [9], pp. 37 and 129), where additionally the condition Equation (72) is to be taken into account.

For metallic phases, the non-electronic contribution to $\kappa_{i}, \kappa_{n e, i}$, can often be neclected compared to $\kappa_{e, i}$, if the carrier densities are not too small. If not, $\kappa$ is to be calculated by Equation (23) under consideration of Equation (24), where the $\kappa_{n e, i}$ are to be determined separately; that becomes especially important for semiconducting phases and if the phase $i$ does not form a macroscopic cluster.

For the Hall coefficient of the phase $i$ in a nonmagnetic composite, $R_{i}$ in NFE-approximation is given by

$$
R_{i}=-\frac{C}{|e| \cdot n_{i}}=\frac{\mu_{i}}{\sigma_{i}}
$$

where $\mu_{i}$ is the Hall mobility of the phase $i$ and $C$ is a parameter of the order of one depending slightly on the magnetic field. [14] [15].

The volume fractions of the phases, $v_{i}$, can be calculated from the atomic concentrations of the composite and the phases, $x$ and $x_{i}$, respectively. For a two-phase composite, the $v_{i}$ can be determined by

$$
v_{A}=1-v_{B}=\left[1+\frac{\mathcal{N}_{A}\left(x-x_{A}\right)}{\mathcal{N}_{B}\left(x_{B}-x\right)}\right]^{-1},
$$

where $\mathcal{N}_{A}$ and $\mathcal{N}_{B}$ are the atomic densities in the phases $A$ and $B$, respectively.

\subsection{Nanocomposites}

\subsubsection{Electron Density in Nanocomposites}

Let us consider the class of amorphous transition-metal-metalloid alloys in more detail: it was one of the most important results that amorphous transition-metalmetalloid alloys do generally not consist of randomly mixed atoms, but they form composites consisting of amourphous phases which differ regarding their short range order (SRO). Interpreting a series of transport data of amorphous transitionmetal-metalloid alloys, Sonntag [32] has drawn the following conclusions:

For large ranges of concentration there is

(i) amorphous phase separation between two different amorphous phases called phase $A$ and phase $B$, where each phase has its "own" short-range order (SRO),

(ii) the amorphous phase separation leads to band separation in the con- 
duction band (CB) and valence band (VB) connected with the phases $A$ and $B$, respectively, and the electrons are freely propagating and the corresponding wave functions are extended with respect to connected phase ranges.

(iii) Between the two coexisting phases there is electron redistribution (electron transfer) which can be described by

$$
n(\zeta)=n_{A} \exp (-\beta \zeta)
$$

where $\zeta$ is the quotient of the volume or atomic fractions of the two coexisting phases. $n(\zeta)$ is the electron density in the phase $A$ with $n_{A}=n(0) . \quad \beta$ is a constant for a given alloy, which is determined by the average potential difference between the two phases.

For a series of amorphous transition-metal-metalloid alloys, conclusion (i) and conclusion (ii) are now confirmed experimentally or supported by independent authors [2] [3] [4] [5] [6] [43] [44] [45] [46] [47]. For details see [33] (Section 1 therein). Although the conclusion (iii) is not yet confirmed or supported by independent authors, there are a series of experimental findings supporting this conclusion (iii), as demonstrated in [32], where for some amorphous transition-metal-metalloid alloys it is shown that $\rho$ versus $x /(1-x)$ follows an exponential concentration dependence (see Figures 1-6 in [32]). For instance, $a-\mathrm{Cr}_{1-x} \mathrm{Si}_{x}$ thin films measured by Helms et al. [39] and shown in Figure 4 follow an exponential dependence of $\rho$ versus $x /(1-x)$. As argued in [32], one can assume that for $x>0.25 \quad a-\mathrm{Cr}_{1-x} \mathrm{Si}_{x}$ thin films consist of the two amorphous phases $a-\mathrm{Cr}_{3} \mathrm{Si}(=$ phase $A$ ) and $a$-Si (=phase B), i.e., the silicon concentrations in the two phases are characterized by $x_{A}=0.25$ and $x_{B}=1$, respectively, whereas for $0 \leq x<0.25$, the films are one-phase. In the two-phase range, $\zeta \quad\left(=X_{B} / X_{A}\right)$ can be calculated by

$$
\zeta=\frac{X_{B}}{X_{A}}=\frac{x-x_{A}}{x_{B}-x}
$$

As can be seen in Figure 4(b) (lower grafic), $\rho$ follows an exponential dependence on $\zeta\left(=X_{B} / X_{A}\right)$. This result is in correspondence with Equation (84) if the effect of $\sigma_{B}$ (about Equation (19)) as well as the effect of a variation of the electronic mean free path $L$ can be neglected. Because the scattering in $a-\mathrm{Cr}_{1-x} \mathrm{Si}_{x}$ is very strong, $L$ is very small. However, $L$ cannot be smaller than the average atomic distance, $d$; therefore one can assume that it is nearly independent of $x$, that is, $d \approx L$ and $\sigma_{A}\left(=1 / \rho_{A}\right)$ is expected to be directly proportional to the electron density, $n$. The effect of the second phase $(a-S i=$ phase $B)$ on $\rho(=1 / \sigma)$ (about Equation (19)) can be neglected, because in ${ }_{a}-\mathrm{Cr}_{1-x} \mathrm{Si}_{x}$ the concentration dependence of $\sigma_{B}$ is similar to $\sigma_{A}$. This conclusion follows from calculations done in [11] (see Figure 4(c) therein).

Support for Equation (84) comes also from Hall coefficient measurements of metal-insulator composites as shown in Figure 5. The sizes of the phase grains are of the order of some atomic distances as well. In Figure 5(a) and Figure 5(b) the experimental original data of $\mathrm{Cu}_{1-y}\left(\mathrm{SiO}_{2}\right)_{y}$ and $\mathrm{Ni}_{1-y}\left(\mathrm{SiO}_{2}\right)_{y}$ taken from [40] 

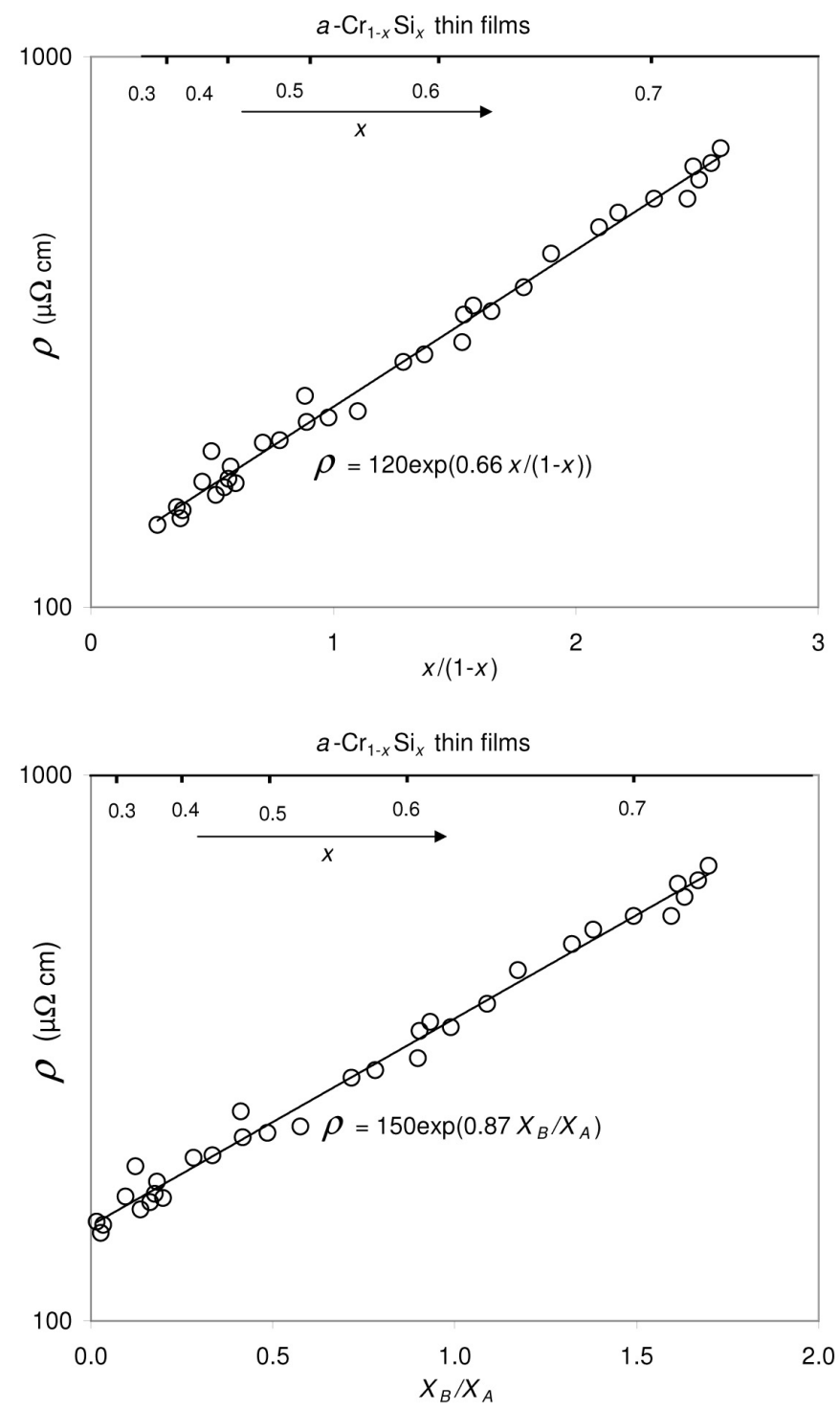

Figure 4. Experimental data $\rho$ versus $x /(1-x)$ (upper diagram) and $\rho$ versus $X_{B} / X_{A}$ (lower diagram) for $a-\mathrm{Cr}_{1-x} \mathrm{Si}_{X}$ thin films at $T=300 \mathrm{~K}$ taken from Helms et al. [39]. $X_{B} / X_{A}$ is calculated by $X_{B} / X_{A}=\left(x-x_{A}\right) /\left(x_{B}-x\right)$ with $x_{A}=0.25$ and $x_{B}=1.00 . X_{B}$ and $X_{A}$ are the atomic fractions of the amorphous phases $a-\mathrm{Cr}_{3} \mathrm{Si}$ (=phase $A$ ) and $a$-Si (=phase $B$ ).

and [41] are drawn versus $y /(1-y)$, and in Figure 5(c) and Figure 5(d) the corresponding $R_{A}$ drawings calculated by Equation (63) ( $\sigma_{B}=0$, because $\mathrm{SiO}_{2}$ is an insulator). The exponential concentration dependence of $R_{A}$ in Figure 5(c) and Figure 5(d) reflects immediately Equation (84) because of Equation (82).

$R$ and $R_{A}$ versus $y /(1-y)$ follow an exponential dependence also for $\mathrm{Ni}_{1-y}\left(\mathrm{SiO}_{2}\right)_{y}$ although we had assumed non-magnetic materials for the derivation of Equation (43), wherease $\mathrm{Ni}$ is a magnetic material. This is however not a 


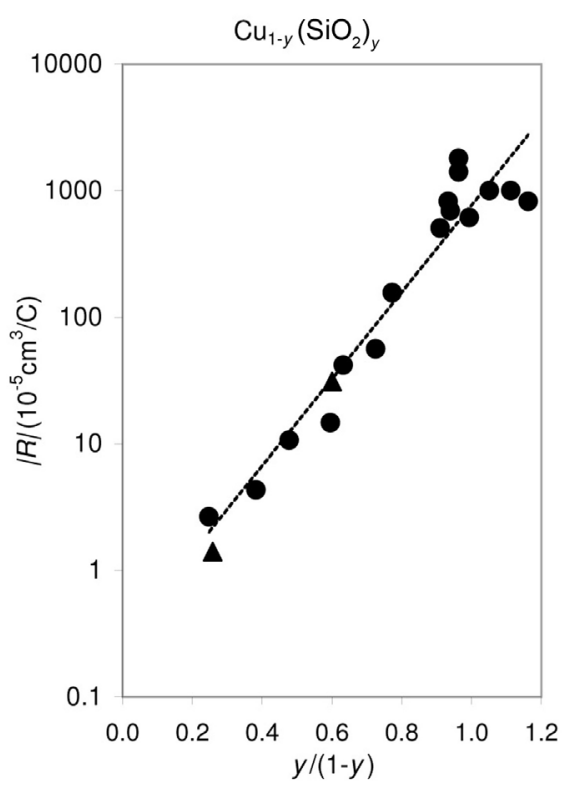

(a)

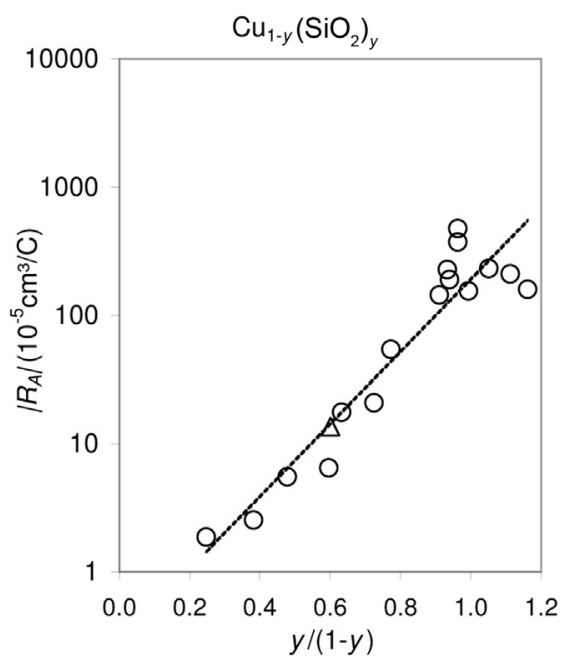

(c)

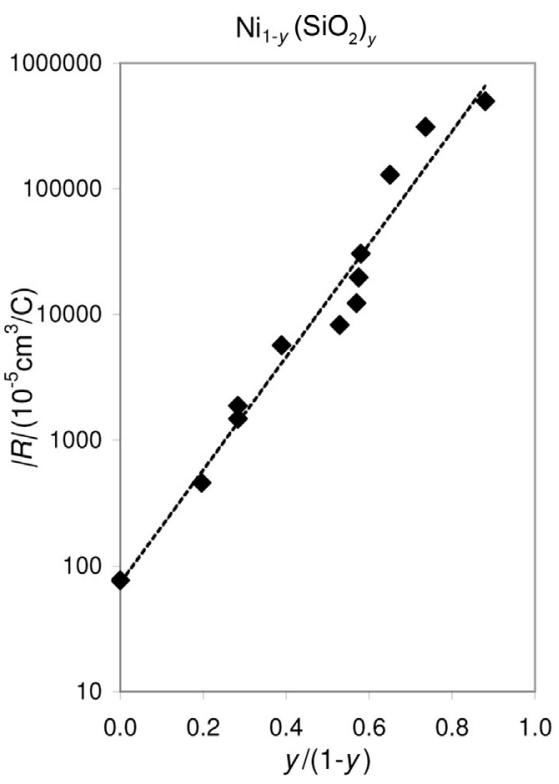

(b)

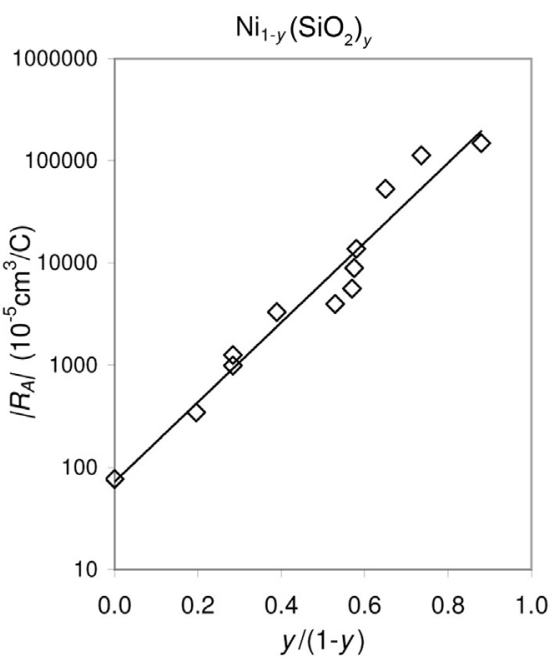

(d)

Figure 5. Experimental Hall coefficient data at $5 \mathrm{~K}$ versus $y /(1-y)$ for $\mathrm{Cu}_{1-y}\left(\mathrm{SiO}_{2}\right)_{y}$ (a), (c), and $\mathrm{Ni}_{1-y}\left(\mathrm{SiO}_{2}\right)_{y}$ (b), (d), taken from [40] (circles), [41] (triangles) and [42] (diamonds). (c), (d): $R_{A}$ calculated by $R_{A}=R \frac{\left(3 v_{A}-1\right)}{2}$ according to Equation (43), where $v_{A}=1-y$ is set.

discrepance. For magnetic metal-insulator composites Equation (82) holds approximately if "=" is replaced by " $\propto$ " considering the effect of the additional internal magnetic field due to the magnetization: An electron sees the effective magnet field $H_{w}=H+H_{i}$, where $H_{i} \gg H . H$ is the external field applied to the specimen and $H_{i}$ is the internal field produced by the quantum mechanical exchange forces ([48], p. 341). An electron does not distinguish between $H$ and $H_{i}$. It moves according to the Lorentz force determined by $H_{w}$ and the electric field $E$. One can assume that $H_{w}$ is nearly proportional to $H$ as long as $H_{i}$ is 
nearly proportional to the magnetization produced by $H$.

This assumption is supported by the experimental finding by Xiong et al. [49] that (for not too small fields $H$ ), in the granular Co-Ag system, the Hall resistivity $\rho_{x y}$ is linearly proportional to $H$. If so, the measured $R$ values differ from the calculated $R$ values, Equation (82), only by a factor which is nearly constant. Therefore, we assume that the EMT formula for $R$, Equation (43), can be applied to magnetic composites as well.

If the metallic phase of a M-I composite is a noble metal, the NFE-approximation is a good one for the metallic phase, above all as the Fermi surface moves away from the Brillouin zone boundary as $n$ decreases. For the metallic phase in $\mathrm{Ni}-\mathrm{SiO}_{2}$ the NFE-approximation is surely also a good one, because $\mathrm{Ni}$ has only $0.554 \mathrm{~s}$ valence electrons per Ni atom ([48], p. 271).

Additional support for Equation (84) comes from experimental resistivity data of amorphous $\left(\mathrm{Cr}_{1-x} \mathrm{Si}_{x}\right)_{1-z} \mathrm{~N}_{z}$ and $\left(\mathrm{Cr}_{1-x} \mathrm{Si}_{x}\right)_{1-y} \mathrm{O}_{y}$ thin films (in preparation).

\section{Amorphous Metals}

The electron transfer $\delta n$ between the phases described by Equation (84) leads to a lowering of the total energy of the composite compared with a situation, where the phases exist alone. This is the reason for the fact that metallic composites with an amorphous structure can exist at all. Almost invariably, amorphous metals contain a metalloid as one of the constituents. e.g., $\mathrm{Au}-\mathrm{Si}, \mathrm{Pd}-\mathrm{Si}, \mathrm{Fe}-\mathrm{P}-\mathrm{C}, \ldots[$... [50]. That is, one-phase amorphous metals practically do not exist. If yet (for instance after an evaporation process at extremely low temperatures), then there is the strong suspicion that during the preparation process impurities as oxygen and nitrogen are incorporated in the metal film.

Of course, for the crystalline state the energy gain is surely larger compared with that of the amorphous state. However, the transition from the amorphous state to the crystalline one realized by atomic diffusions processes requires additional energy to overcome energy barriers.

\section{The Giant Hall Effect (GHE)}

As reasons for the very large values of the Hall coefficient in metal-insulator composites as shown in Figure 5 for the examples $\mathrm{Cu}_{1-y}\left(\mathrm{SiO}_{2}\right)_{y}$ and $\mathrm{Ni}_{1-y}\left(\mathrm{SiO}_{2}\right)_{y}$ (known as the "Giant Hall effect"), quantum size effects and quantum interference effects on the mesoscopic scale have been discussed. [40] [51] [52] [53] [54] [55] However, to our knowledge, until now, there is no explanatory model which can interpret the phenomenon of GHE. Now, we believe that Equation (84) gives a simple and plausible explanation for the GHE.

\subsubsection{Electronic Transport in Nanocomposites}

For many alloys with phase separation the phase grains are very small so that an application of the classical transport equations to the phase grains (Section 3.1) does not seem to be appropriate. In spite of this reservation, both the BTE and the approximation of free electrons (NFE-approximation) may be good descriptions for a phase $i$ of a composite, as long as it forms an infinite cluster in the composite and the scattering processes are elastic. For amorphous transition- 
metal-metalloid alloys this condition is fulfilled for $v_{i}>1 / 3$. This point of view is justified in [33] (Section 4.2 therein) and [12] (Section 5.2. therein). That is, formulas for the transport coefficients of the phases, Equations (79)-(82), may be a good description if the phase $i$ forms a macroscopic cluster through the composite corresponding to $v_{i}>1 / 3$. For this case the phase $i$ can form a quasi-continuous energy band and the scattering is elastic. With decreasing $v_{i}$, the macroscopic $i$ phase cluster decomposes into separate phase grains; for amorphous composites, this transition occurs very precisely at $v_{i}=1 / 3$. The reasons are the following [12] [33]:

(1) As the grain diameters are very small (of the order of $\sim 1-2 \mathrm{~nm}$ [5] [6], the number of grains (of the same sort $i$ ) must be very large to form a cluster for which a quasi-continuous energy band is realized. Accidentially formed agglomerates of some (for instance 10 or 100 or 1000) neighbouring grains (with $\sim 1-2 \mathrm{~nm}$ diameter) are not sufficient for forming a quasi-continuous energy band.

(2) During the film deposition of a composite, the atoms of the different atom sorts arrive at the substrate equally distributed; therefore the different phase grains $(A$ and $B$ ) can also be assumed to be locally equally distributed in the amorphous composite, because the diffusion paths during solidification are very short, which is a prerequisite for forming amorphous composites.

A phase is an "electronic phase" determined by a solution of the Schrödinger equation; after hitting at the substrate, the atoms move locally only so long until they can form a phase which corresponds to a solution of the Schrödinger equation. That is why, the phase grains of the same sort $i$ are also locally equally distributed, as the compositions of the different phases are very different, i.e., the local distribution of the $i$ phase grains is not completely random, as, e.g., assumed within the framework of the classical percolation theory. For such a locally equally distributed arrangement of the $i$ phase grains in an amorphous matrix (formed by the rest of the composite), it follows that this merging to a macroscopic cluster through the sample occurs very precisely at a specific concentration $v_{i}$; and this specific concentration is $v_{i}=1 / 3$, as follows, for instance, from Equation (19) setting $\sigma_{j}=0$, but $\sigma_{i} \neq 0 \quad(j \neq i)$. (For a comparison with the classical percolation theory concerning this critical value of $v_{i}$, see also [33] (Section 4.1 therein).

(3) A macroscopic $i$ phase cluster is only realized, if all the atoms in this $i$ phase cluster are directly connected to atoms belonging to the same phase sort $i$. When, for instance, two grains of the same phase sort $i$ are separated by a monoatomic layer of a different(!) phase $(j)$, these two $i$ phase grains cannot be considered as (nearly) one $i$ phase cluster, because the overlap of the electron wave functions is interrupted by this monoatomic layer. Within an $i$ phase grain or $i$ phase cluster the wave functions, $\psi_{i}(r)$, overlap, but they do not overlap between two $i$ phase grains or $i$ phase clusters separated by a monoatomic layer of a different(!) phase ( $j)$. 
If a sufficiently large number of $i$ phase grains form a macroscopic cluster, the overlapping wave functions $\psi_{i}(r)$ form a quasi-continuous energy band, while the wave functions fall off exponentially in a very short distance $|\delta r|$ outside this macroscopic cluster. This "falling off" is comparable with the decrease of the molecular orbitals of (large) molecules at their molecule boundaries (see, e.g., [56], pp. 409 and pp. 435, or [57]). Because of this "non-overlapping" of the $\psi(r)$ belonging to different phases, they do not "penetrate" each other, i.e., there are sharp boundaries between the different (electronic) phases.

Considering the fact that the phase $i$ does not form a quasi-continuous energy spectrum for $v_{i}<1 / 3$, but there is a discrete energy spectrum typical for separate grains, then there are no electronic states immediately above and below $\tilde{\mu}$ (within the energy range $k_{B} T$ ), i.e., the electrons cannot be activated to higher energies (at the hot end of the sample) and cannot deliver energy (at the colder end), if $v_{i}<1 / 3$. Under this condition it follows that (at the temperature T) $\kappa_{e, i}=0$ for $v_{i}<1 / 3$. For $S_{i}$ the situation is analogous: for $v_{i}<1 / 3$, $S_{i}^{0}=0$, i.e., at the transition from $v_{i}>1 / 3$ to $v_{i}<1 / 3$, both $\kappa_{e, i}$ and $S_{i}$ change discontinuously. Such discontinuities are especially to be expected in composites with metallic phases.

For amorphous transition-metal-metalloid alloys, " $\kappa_{e, i}=0$ " and " $S_{i}^{0}=0$ " for $v_{i}<1 / 3$ is only an approximation, because, at the boundary faces between the different phases, there are $p$ - $d$ bonds, i.e., $d$ orbitals of the $\mathrm{Cr}$ atoms (of the $A$ phase grains) overlap with $p$ orbitals of the boundary faces atoms on the $B$ phase grains resulting to a $p-d$ band, which is incompletely occupied. (For a detailed discussion see Section 2.1 of [33]). Because of this $p$ - $d$ overlapping, this (incompletely occupied) $p$ - $d$ band exists also if $v_{A}<1 / 3$ : although the energy levels of the $s$ states in the separate $A$ phase grains are discret, the electrons can be activated to higher energies (at the hot end of the sample) and deliver energy (at the colder end) by a transition to the $p$ - $d$ band. That is why, $\kappa_{e, A} \neq 0$ and $S_{A}^{0} \neq 0$, also for $v_{A}<1 / 3$. However, the real values of $\kappa_{e, A}$ and $S_{A}^{0}$ (for $v_{A}<1 / 3$ ) are expected to be essentially smaller than calculated by Equations (80) and (81), because the $d$ states are localized.

\section{Discontinuity in the Concentration Dependence of the Thermopower}

A specific feature of composites with $S_{A}>0$ and $S_{B}<0$ or vice versa is the fact that a discontinuity (step) in the calculated $S$ vs. $v_{i}$ can occur, which is an additional possibility to check experimentally the thermopower formula. One example for a composite with different signs of $S_{A}$ and $S_{B}$ is $a-\mathrm{Cr}_{1-x} \mathrm{Si}_{x}$ consisting of the two amophous phases [33] $a-\mathrm{Cr}_{1-x_{A}} \mathrm{Si}_{x_{A}}$ and $a-\mathrm{Cr}_{1-x_{B}} \mathrm{Si}_{x_{B}}$ with $x_{A} \approx 0.25$ and $x_{B} \approx 1$. (Amorphous phase separation is typical for many amorphous transition-metal-metalloid alloys [2] [3] [4] [5] [6]). In [12] $S$ versus $x$ had been calculated for $a_{-}-\mathrm{Cr}_{1-x} \mathrm{Si}_{x}$ applying Equation (31) for $x_{A}=0.25$ and $x_{B}=0.90 \quad$ (bold line in Figure 1 of [12]). 
Now, the calculations of [12] we have done once more, but for $x_{A}=0.25$ and $x_{B}=1.00$ shown in Figure 6 commonly with the result of the calculations for $x_{A}=0.25$ and $x_{B}=0.90$. Both the discontinuity (step) at $x=0.49$ and the general trend of the calculated curves agree with the experimental data.

The discontinuity of $S$ versus $v_{i}$ for composites with $S_{A}>0$ and $S_{B}<0$ or vice versa has its origin in the mathematic structure of the formula

$$
S( \pm)=\frac{4 \cdot \kappa_{e}}{\frac{\kappa_{e, A}}{S_{A}}\left(3 v_{A}-1\right)+\frac{\kappa_{e, B}}{S_{B}}\left(3 v_{B}-1\right) \pm \sqrt{\left(\frac{\kappa_{e, A}}{S_{A}}\left(3 v_{A}-1\right)+\frac{\kappa_{e, B}}{S_{B}}\left(3 v_{B}-1\right)\right)^{2}+8 \frac{\kappa_{e, A} \cdot \kappa_{e, B}}{S_{A} \cdot S_{B}}}}
$$

following from Equation (31) for two-phase composites, where $v_{B}=1-v_{A}$. Equation (86) has two solutions, $S(-)$ and $S(+)$, which both show a discontinuity (step) at the same concentration, when $S(-)$ and $S(+)$ passes the value " 0 " coming from negative values crossing to positive values or vice versa. The physics follows only one of them, $S(-)$, as suggested by the results of [11] (compare Figure 4(g) and Figure 4(h) with Figure 5 therein).

As this discontinuity occurs at $S=0$, this phenomenon opens the possibility

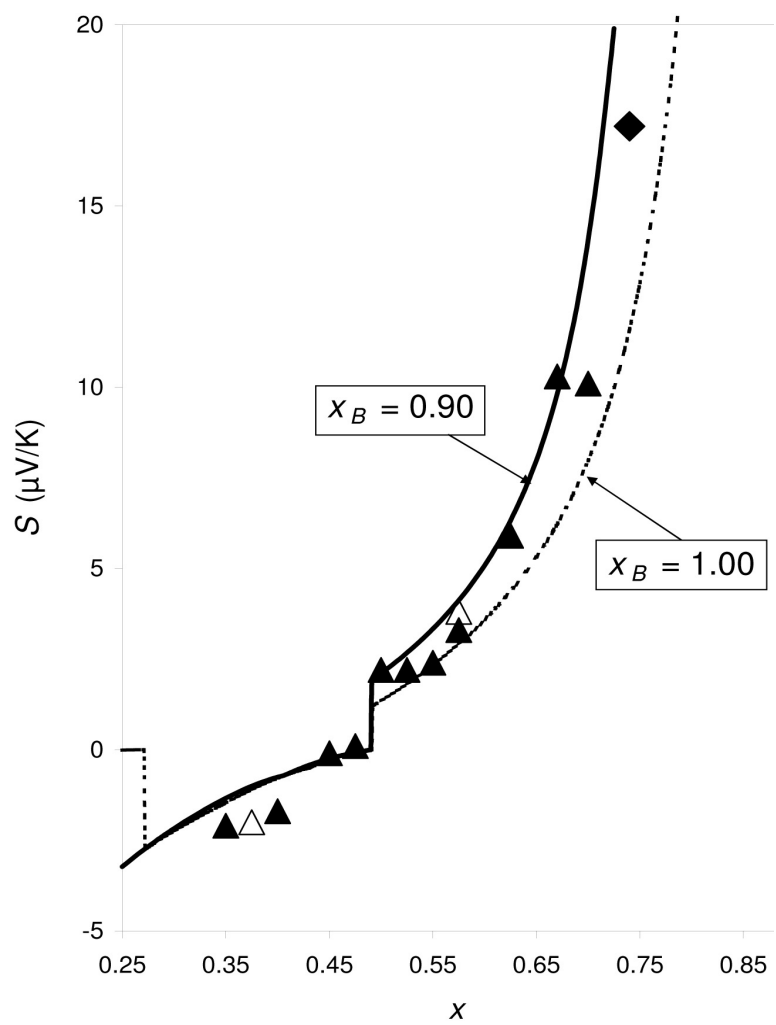

Figure 6. Thermopower versus $x$ for $a-\mathrm{Cr}_{1-x} \mathrm{Si}_{x}$ at $T=300 \mathrm{~K}$ calculated by Equation (32) with $x_{A}=0.25$ and $x_{B}=0.90$ (bold line) and $x_{A}=0.25$ and $x_{B}=1.00$ (dotted line) and comparison with experimental data. Details of the calculations and experimental data are described in [11] (section IVB therein). 
to produce reference standards for absolute thermopower $S=0$ even for $T>T_{c}$ ( $T_{c}$ stands for the transition temperature of any superconductor).

\section{Extension of the Classical Thermopower Formula}

In the limit $v_{B}=0$, the composite degenerates to a homogeneous alloy consisting exclusively of the phase $A$. On the opposite side, for $v_{B}=1$ we get a homogeneous alloy consisting exclusively of the phase $B$. For these two limiting cases the formulae must hold as well. Setting $v_{B}=0$, it follows from Equation (74) that

$$
\frac{\mathrm{d} \tilde{\mu}}{\mathrm{d} T}=\frac{\partial E_{C, A}}{\partial T}+\frac{\partial \tilde{\mu}_{A}^{0}}{\partial T}
$$

and taking into account Equations (73), (81), (68) and Equation (22),

$$
S(+)=S_{A}=\frac{\pi^{2} k_{B}^{2} T\left(1+r_{A}\right)}{3 e_{A} E_{F, A}}+\frac{1}{|e|}\left(\frac{\partial E_{C, A}}{\partial T}+\frac{\partial \tilde{\mu}_{A}^{0}}{\partial T}\right) .
$$

Analogously it follows for $v_{B}=1$ that

$$
\begin{gathered}
\frac{\mathrm{d} \tilde{\mu}}{\mathrm{d} T}=\frac{\partial E_{V, B}}{\partial T}-\frac{\partial \tilde{\mu}_{B}^{0}}{\partial T} \\
S(+)=S_{B}=\frac{\pi^{2} k_{B}^{2} T\left(1+r_{B}\right)}{3 e_{B} E_{F, B}}+\frac{1}{|e|}\left(\frac{\partial E_{V, B}}{\partial T}-\frac{\partial \tilde{\mu}_{B}^{0}}{\partial T}\right) .
\end{gathered}
$$

Inserting Equation (75) in Equations (88), (90) we get

$$
\begin{gathered}
S=-\frac{\pi^{2} k_{B}^{2} T(z+r)}{3|e| E_{F}}+\frac{1}{|e|} \frac{\mathrm{d} E_{C}}{\mathrm{~d} T}, \\
S=\frac{\pi^{2} k_{B}^{2} T(z+r)}{3|e| E_{F}}+\frac{1}{|e|} \frac{\mathrm{d} E_{V}}{\mathrm{~d} T},
\end{gathered}
$$

with $z=3 / 2$, where the index $i$ is omitted and $\partial E_{C, A} / \partial T$ and $\partial E_{V, B} / \partial T$ are replaced by $\mathrm{d} E_{C} / \mathrm{d} T$ and $\mathrm{d} E_{V} / \mathrm{d} T$, respectively, because a homogeneous metal consists only of one phase. Each of the two limiting cases, Equation (91) and Equation (92), represents a homogeneous metal with electron conductivity and hole conductivity, respectively, and constant carrier density, i.e., interband transfer of electrons (in dependence on temperature) are not considered.

The term " $\partial \tilde{\mu}_{i}^{0} / \partial T$ " reflects the lowering of the chemical potential with $T$ described by the Fermi-Dirac-statistics. In Equations (87)-(92) it is assumed that Equation (81) does exclusively represent the scattering part of $S$. It is, however, not completely clear, whether Equation (81) does indirectly contain this term “ $\partial \tilde{\mu}_{i}^{0} / \partial T$ ”, already. If so, then in Equations (88), (90)-(92) the term “ $\partial \tilde{\mu}_{i}^{0} / \partial T$ ” (respectively “ $-\frac{\pi^{2} k_{B}^{2} T}{6 E_{F, i}}$ ”) is to be deleted, and the value for $z$ is to be replaced by $z=1$.

Equation (81) agrees with the first term in Equation (91) if $z=1$ is set. It represents the contribution of the scatterring on the thermopower, whereas the 
second term in Equation (91) represents the effect of the temperature dependance of the band edge on the thermopower. Therefore, we can say that for normal metals positive sign of thermopower will be measured if $\mathrm{d} E_{C} / \mathrm{d} T>0$ and if this effect overcompensates for the influence of the first term in Equation (91). This conclusion holds exactly if $z=1$. If $z=3 / 2$, this fact is to be considered as a tendency.

As mentioned earlier, it is not yet completely clear whether $z=3 / 2$ or $z=1$. We believe that for metals $z=3 / 2$ is correct. However, the final answer depends on the question whether or not Equation (81) contains exclusively the scattering contribution [corresponding to $S^{0}$, Equation (81)]. This question is a matter of future studies.

\section{Simple Metals with Positive Thermopower}

It is a widely forgotten mystery, why the thermopower of a metal as simple as lithium is positive [10] [58] [59] [60] [61]. Considering only the scatterring term of Equation (91) (as it was classical standard theory according to Equation (81)), positive sign was incomprehensible. However, considering the second term of Equation (91), this puzzle is now solved. Positive sign of thermopower can also be expected for crystalline metals: As a trend, positive sign of thermopower will be measured, if the conduction band edge shifts to higher values with increasing $T$ and if this effect overcompensates for the influence the scatterring term. Thus, for the crystalline $\mathrm{Cu}, \mathrm{Ag}, \mathrm{Au}$, and $\mathrm{Li}$ the experimental thermopowers also have positive sign; at $0^{\circ} \mathrm{C}$ they are $+1.7,+1.4,+1.1$, and $+11.5 \mu \mathrm{V} / \mathrm{K}$, respectively ([48], p. 316). For more details see [38].

\section{Constraints of the Combinatorial Development of Materials}

The combinatorial development of materials is a very effective method to get experimental data about a certain material system, because a large range of different compositions can be realized concurrently on one large substrat, for instance by deposition of thin films by simultaneous co-sputtering from two or three targets on a large substrat. On the other hand, this method is to be used with great caution because the results can be completely different from a situation where the different compositions are produced separated in single manufacturing processes. The reasons are the following ones.

In a composite a common electrochemical potential is realized by electron transfer between the different phases. This can lead to the fact that in one of the phases another topological structure (atomic configuration) is more favorable than for the case that this phase exists alone (as homogeneous material). And in nanocomposites the electron transfer has an essential larger effect on the carrier densities and therefore on the transport coefficients in the phases compared with composites with large phase grains.

Figure 7 shows two series of resistivity measurements of $\mathrm{Cr}_{1-x} \mathrm{Si}_{x}$ thin films 


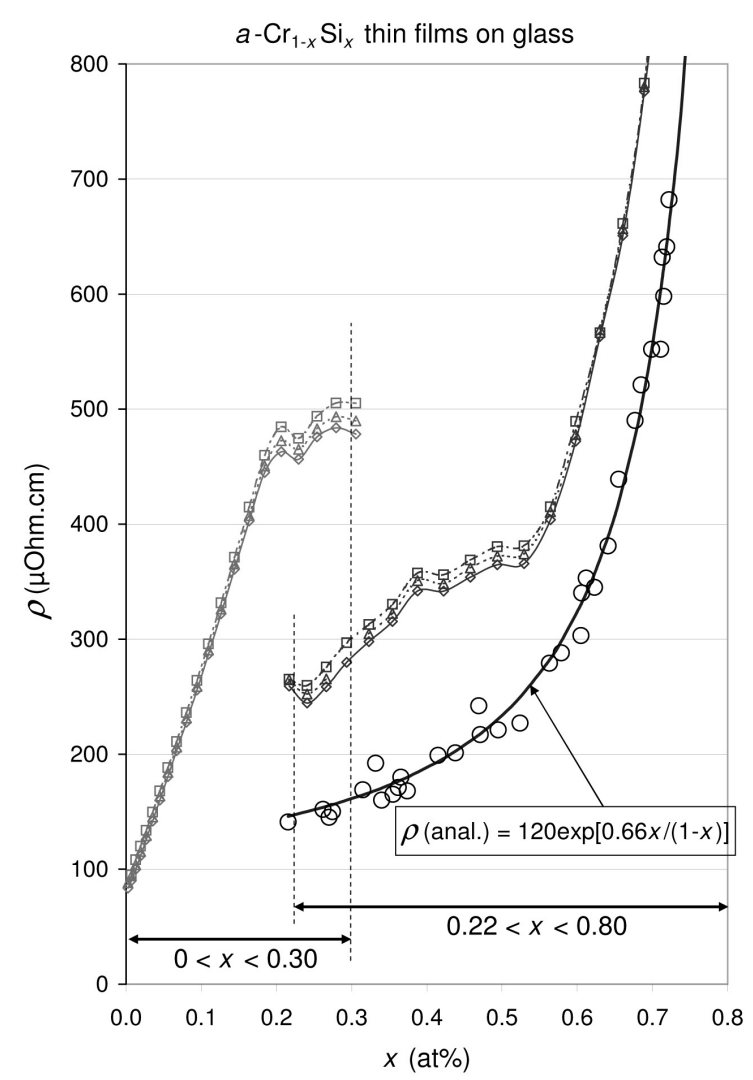

Figure 7. Experimental resistivity data of $\mathrm{Cr}_{1-x} \mathrm{Si}_{x}$ thin films cosputtered from a chromium target and a silicon target. By variation of the sputtering rates two different concentration ranges about 4 inch glass wafers are realized, 1) $0<x<0.30$, and 2) $0.22<x<0.80$. Details of film deposition, analysis and resistivity measurements are described in [62]. Additionally the resistivity data of co-sputtered $\mathrm{Cr}_{1-x} \mathrm{Si}_{x}$ thin films by Helms et al. [39] are shown (circles; already shown as a logarithmic representation in Figure 4), where the single samples were separated from each other during the deposition process.

cosputtered from a chromium target and a silicon target and deposited on 4 inch glass wafers (the deposition conditions are specified in [62] [63]). Three parallely lying measuring lines have been measured with a distance of $4.5 \mathrm{~mm}$ to each other. The first series extend continuously about the concentration range $0<x<0.30$, the second one about $0.22<x<0.80$. The corresponding film thickness were $200 \mathrm{~nm}>d>80 \mathrm{~nm}$ and $165 \mathrm{~nm}>d>131 \mathrm{~nm}$, respectively. Additionally resistivity data received from Helms et al. [39] are drawn. These data were produced by co-sputtering on glass as well, but with the difference that each measuring point represent a single sample; these single samples were separated from each other during the deposition process, i.e., there were no electrical connections between the single samples. The structure of all the films were detected to be X-ray amorphous, where for the chromium rich samples microcristalline precipitations has been found; number and average size of these 
precipitations decrease with the silicon content.

As can be seen in Figure 7, in the overlapping range between the two series " $0<x<0.30$ " and " $0.22<x<0.80$ ", there are very large $\rho$ differences, nearly a factor of $1.7 \ldots 1.8$. The factor between the " $0<x<0.30$ " series and the Helms samples is even 3.0 ... 3.1.

Figure 8 shows experimental thermopower data of the same samples as shown in Figure 7 supplemented by a third series for the concentration range $0.49<x<0.96$ with the corresponding film thickness of $70 \mathrm{~nm}<d<260 \mathrm{~nm}$ [62]. The structures were detected to be X-ray amorphous. $S$ has been measured applying the Potential-Seebeck Microprobe (PSM) from the company LOT-Oriel Group Europe [64] [65].

The $S$ curves for the concentration ranges $0<x<0.30$ and $0.22<x<0.80$ as well as for the single samples (triangles) correspond relatively well. However, between the second series and the third one there are large differences. The discontinuity at $S=0$ occurring in the $S$ curve of the single samples does not occur in the co-sputtered samples.

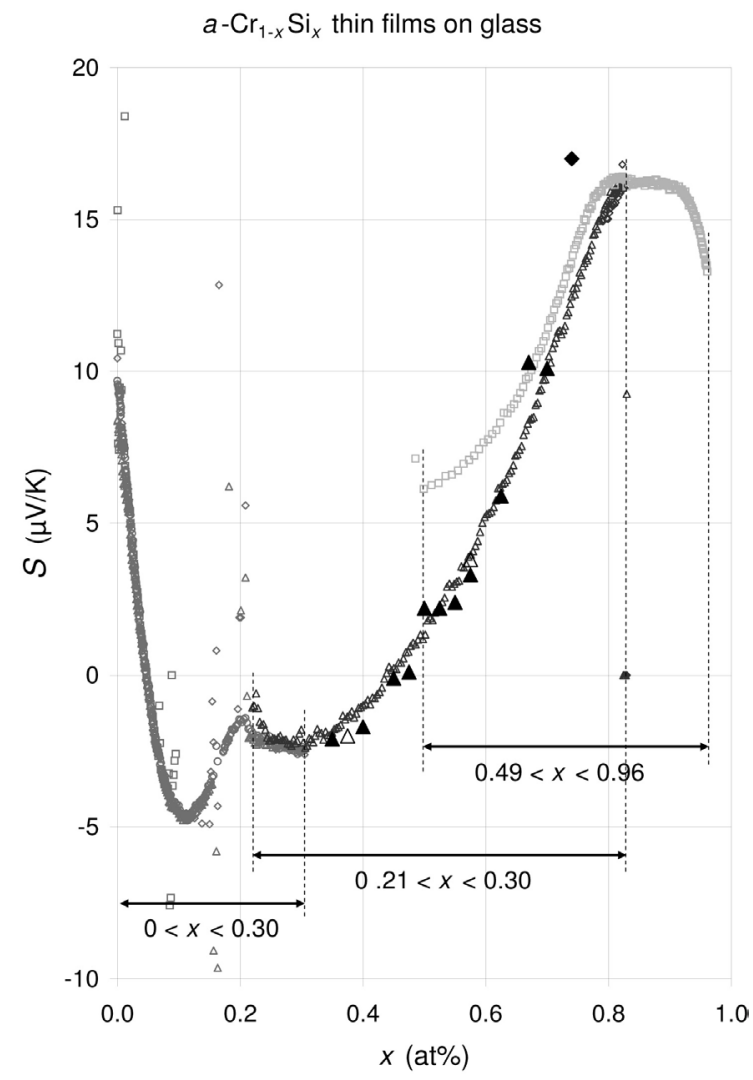

Figure 8. Experimental thermopower data of $\mathrm{Cr}_{1-x} \mathrm{Si}_{x}$ thin films for the same samples as shown in Figure 7 supplemented by a third series $0.49<x<0.96$. Details of film deposition, analysis and thermopower measurements are described in [62]. Additionally it is drawn the experimental $S$ data of the $\mathrm{Cr}_{1-x} \mathrm{Si}_{x}$ thin films shown in Figure 6 already. 
Figure 9 shows thermopower data of $a-\left(\mathrm{Cr}_{1-x} \mathrm{Si}_{x}\right)_{1-y} \mathrm{O}_{y}$ thin films co-sputtered as described in [62] for $\mathrm{Cr}_{1-x} \mathrm{Si}_{x}$ thin films, where additionally an oxygen gradient was realized, lateral and perpendicular to the wafer. $S$ has been measured at the complete wafer and after that the wafer was sawed producing single $8 \times 2 \mathrm{~mm}^{2}$ pieces. Now $S$ was measured once more at these pieces. The $S$ vs. $x /(1-x)$ curves for the "wafer" and the "pieces" are shifted nearly parallely to each other, where the zero-crossing of $S$ is shifted from $x=0.31$ to $x=0.59$ (corresponding to $x /(1-x)=0.45$ to $x /(1-x)=1.45$.

The experimenal results shown in Figures 7-9 demonstrate strikingly that the combinatorial development of materials can provide results which can be different from samples produced by single procedures with a given composition.

\section{Conclusions}

Formulas have been presented for calculation of $\sigma, \kappa, S$, and $R$ in composites. We have shown that these formulas can also be applied successfully to nanocomposites if certain conditions are taken into account, especially the

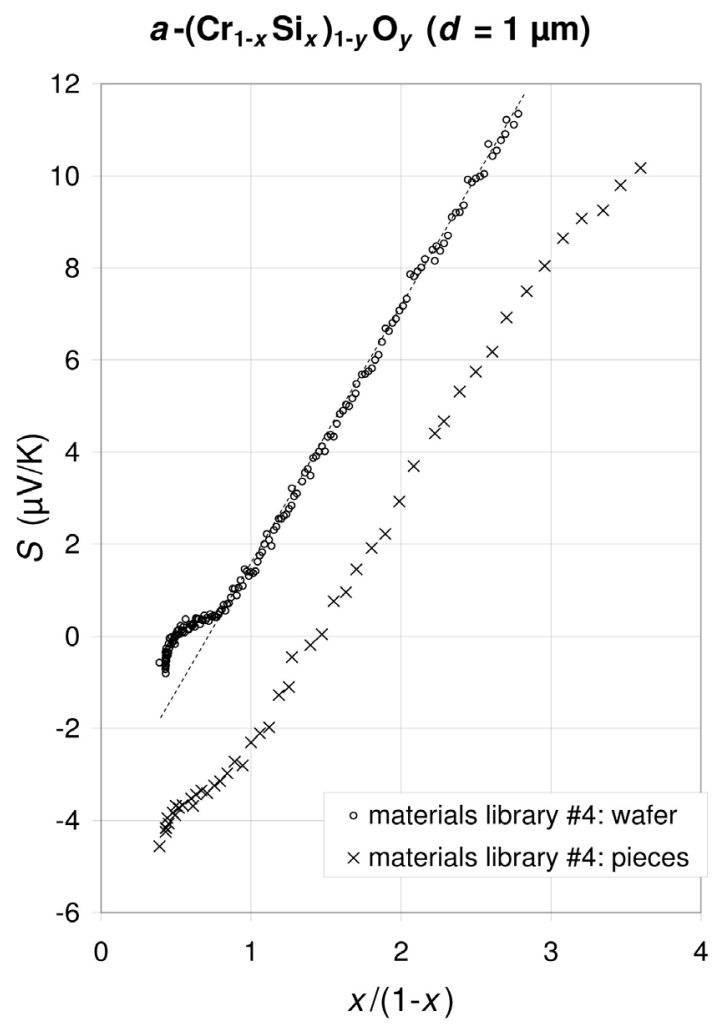

Figure 9. $S$ vs. $x /(1-x)$ of a $a-\mathrm{Cr}_{1-x} \mathrm{Si}_{x}-\mathrm{O}$ thin film with an additional $\mathrm{O}$ gradient vertical to the wafer surface as well as in the same direction as those of silicon concentration gradient (measured for the complete wafer and the pieces after sawing to $8 \times 2 \mathrm{~mm}^{2}$ pieces). The measuring positions with respect to the wafer, before and after sawing, were the same ones. This figure is taken from [62]. 
phenomenon of electron transfer between the different phases in the nanocomposite. It is argued that, under certain conditions, an noncritically application of the combinatorial development of materials can provide unfeasible results.

The formulas and the theory described in the present article give answers to some mysterious puzzles, for which the scientific research had no final answers:

1) Why there are simple metals with positive thermopower?

2) What is the reason for the phenomenon of the "Giant Hall effect"?

3) What is the reason for the fact that amorphous metals can exist at all?

4) Until to the end of the twentieth century amorphous metallic alloys were assumed to be a random and homogeneous distribution of the metal atoms in the amorphous matrix. On this basis, a quantitative calculation applying classic theories was not successful.

The answers given in the present article are the following:

1) The reason for positive thermopower of some metals comes from the temperature dependance of the band edge: If $\mathrm{d} E_{C} / \mathrm{d} T>0$ and if this effect overcompensates for the influence of the scattering term in the thermopower formula, Equation (91), then the thermopower is positive.

2) The reason for the phenomenon of the "Giant Hall effect" in metalinsulator composites is the exponential reduction of the electron density in the metallic phase due to electron transfer from the metallic phase in direction to the insulating phase, described by Equation (84). The transferred electrons are pinned at the phase boundaries between the phases.

3) The reason for the fact that amorphous metals can exist is an electron transfer between the phases described by Equation (84). This electron transfer leads to a lowering of the total energy of the composite compared with a situation, where the phases exist alone. (Amorphous metals are generally composites.)

4) Experimental and theoretical studies at amorphous transition-metal-metalloid alloys have shown that in these amorphous alloys there exists amorphous nano-scaled phase separation between two different amorphous phases, where each phase has its "own" short-range order (SRO) and each phase may be described by its "own" band structure. Within this framework the formulas for $\sigma, \kappa, S$, and $R$ described in this paper can be applyed.

\section{Acknowledgements}

The authors thank Mr. Alan Savan from the Institute of Materials, Faculty of Mechanical Engineering of the Ruhr-University Bochum, and Mr. Michael Kieschnik from RUBION, Ruhr-University Bochum, for co-sputtering the $\mathrm{Cr}$-Si thin films and the RBS analysis, respectively, shown in Figures 7-9.

\section{Conflicts of Interest}

The authors declare no conflicts of interest regarding the publication of this paper. 


\section{References}

[1] Minnich, A.J., Dresselhaus, M.S., Ren, Z.F. and Chen, G. (2009) Bulk Nanostructured Thermoelectric Materials: Current Research and Future Prospects. Energy \& Environmental Science, 2, 466-479.

[2] Edwards, A.M., Fairbanks, M.C., Singh, A., Newport, R.J. and Gurman, S.J. (1989) An Investigation of the Structure of Amorphous $\mathrm{Si}_{1-\mathrm{x}} \mathrm{Ni}_{\mathrm{x}}$ through the Metal-Insulator Transition. Physica B, 158, 600-601. https://doi.org/10.1016/0921-4526(89)90402-X

[3] Edwards, A.M., Fairbanks, M.C. and Newport, R.J. (1991) Structural Studies of Amorphous Ge-Au Alloys. Philosophical Magazine B, 63, 457. https://doi.org/10.1080/13642819108205950

[4] Lorentz, R.D., Bienenstock, A. and Morrison, T.I. (1994) Structural Studies of the Phase Separation of Amorphous FexGe $\mathrm{100- \textrm {x }}$ Alloys. Physical Review B, 49, 3172. https://doi.org/10.1103/PhysRevB.49.3172

[5] Regan, M.J., Rice, M., FernandezvanRaap, M.B. and Bienenstock, A. (1994) Anisotropic Phase Separation through the Metal-Insulator Transition in Amorphous Alloys. Physical Review Letters, 73, 1118. https://doi.org/10.1103/PhysRevLett.73.1118

[6] van Raap, M.B.F., Regan, M.J. and Bienenstock, A. (1995) Evidence of Phase Separation in Amorphous FexSi $i_{1-\mathrm{x}}$ Films. Journal of Non-Crystalline Solids, 191, 155 163. https://doi.org/10.1016/0022-3093(95)00286-3

[7] Odelevskii, V.I. (1951) Raschet obobshchennoy provodimosti geterogennykh system. Zhurnal tekhnicheskoy fiziki, 21, 678.

[8] Landauer, R. (1952) The Electrical Resistance of Binary Metallic Mixtures. Journal of Applied Physics, 23, 779. https://doi.org/10.1063/1.1702301

[9] Harman, T.C. and Honig, J.M. (1967) Thermoelectric and Thermomagnetic Effects and Applications. McGraw-Hill Book Company, New York, San Francisco, Toronto, London, Sydney.

[10] Barnard, R.D. (1972) Thermoelectricity in Metals and Alloys. Taylor and Francis, London.

[11] Sonntag, J. (2006) Disordered Electronic Systems. III. Thermoelectric Power in Alloys with Phase Separation. Physical Review B, 73, Article ID: 045126. https://doi.org/10.1103/PhysRevB.73.045126

[12] Sonntag, J. (2009) Thermoelectric Power in Alloys with Phase Separation (Composites). Journal of Physics: Condensed Matter, 21, Article ID: 175703. https://doi.org/10.1088/0953-8984/21/17/175703

[13] Czycholl, G. (2008) Theoretische Festkörperphysik. Springer Verlag, Berlin, Heidelberg.

[14] Kirejew, P.S. (1974) Physik der Halbleiter. Akademie-Verlag, Berlin.

[15] Kirejew, P.S. (1978) Semiconductor Physics. Mir Publishers, Moscow.

[16] Sonntag, J. (2016) The Origin of the Giant Hall Effect in Metal-Insulator Composites. Open Journal of Composite Materials, 6, 78-90.

[17] Airapetiants, C.V. (1957) Thermal Electromotive Force and Additional Thermal Conductivity of Statistical Mixtures. Soviet Physics Uspekhi, 2, 429.

[18] Webman, I., Jortner, J. and Cohen, M.H. (1977) Thermoelectric Power in Inhomogeneous Materials. Physical Review B, 6, 2959-2964. https://doi.org/10.1103/PhysRevB.16.2959

[19] Halpern, V. (1983) The Thermopower of Binary Mixtures. Journal of Physics C: 
Solid State Physics, 16, L217-L220. https://doi.org/10.1088/0022-3719/16/7/002

[20] Balagurov, B.Y. (1986) Theory of Thermoelectric Properties of Two-Component Media. Soviet Physics Uspekhi, 20, 805.

[21] Bergman, D.J. and Levy, O. (1991) Thermoelectric Properties of a Composite Medium. Journal of Applied Physics, 70, 6821-6833.

https://doi.org/10.1063/1.349830

[22] Hurvits, G., Rosenbaum, R. and Mclachlan, D.S.J. (1993) A Quantitative Analysis of the Thermoelectric Power Measurements on Composite Al-Ge Films. Journal of Applied Physics, 73, 7441. https://doi.org/10.1063/1.353987

[23] Hurvits, G., Rosenbaum, R. and Mclachlan, D.S. (1994) A Quantitative Analysis of the Thermoelectric Power Measurements on Composite Al-Ge Films. Physica A, 207, 391-395. https://doi.org/10.1016/0378-4371(94)90401-4

[24] Herring, C. (1960) Effect of Random Inhomogeneities on Electrical and Galvanomagnetic Measurements. Journal of Applied Physics, 31, 1939-1953.

https://doi.org/10.1063/1.1735477

[25] Fishchuk, I. (1990) Thermomagnetic Properties of Randomly Inhomogeneous Solid-State Systems in Weak Magnetic Fields. Soviet Physics, Solid State, 32, 2053.

[26] Xia, T.K. and Zeng, X.C. (1987) The Effective-Medium Approximation for the Thermoelectric Power of Polycrystals: Application to a Model for $\mathrm{La}_{2-x} \mathrm{M}_{x} \mathrm{CuO}_{4}$. Journal of Physics C: Solid State Physics, 20, L907. https://doi.org/10.1088/0022-3719/20/32/006

[27] Cohen, M.H. and Jortner, J. (1973) Effective Medium Theory for the Hall Effect in Disordered Materials. Physical Review Letters, 30, 696-698.

https://doi.org/10.1103/PhysRevLett.30.696

[28] Cohen, M.H. and Jortner, J. (1974) The Inhomogeneous Transport Regime and Metalnonmetal Transitions in Disordered Material. Journal de Physique, 35, C4345-C4-366.

[29] Vaney, J.-B., Piarristeguy, A., Ohorodniichuck, V., Ferry, O., Pradel, A., Alleno, E., Monnier, J., Lopes, E.B., Goncalves, A.P., Delaizir, G., et al. (2015) Effective Medium Theory Based Modeling of the Thermoelectric Properties of Composites: Comparison between Predictions and Experiments in the Glass-Crystal Composite System $\mathrm{Si}_{10} \mathrm{As}_{15} \mathrm{Te}_{75}-\mathrm{Bi}_{0.4} \mathrm{Sb}_{1.6} \mathrm{Te}_{3}$. Journal of Materials Chemistry C, 3, Article ID: 11090. https://doi.org/10.1039/C5TC02087E

[30] McLachlan, D.S. (1987) An Equation for the Conductivity of Binary Mixtures with Anisotropic Grain Structures. Journal of Physics C: Solid State Physics, 20, 865.

[31] McLachlan, D.S., Blaszkiewicz, M. and Newnham, R.E. (1990) Electrical Resistivity of Composites. Journal of the American Ceramic Society, 73, 2187-2203.

[32] Sonntag, J. (1989) Disordered Electronic Systems: Concentration Dependence of the DC Conductivity in Amorphous Transition-Metal-Metalloid Alloys (Metallic Regime). Physical Review B, 40, 3661-3671. https://doi.org/10.1103/PhysRevB.40.3661

[33] Sonntag, J. (2005) Disordered Electronic Systems. II. Phase Separation and the Metal-Insulator Transition in Metal-Metalloid Alloys. Physical Review B, 71, Article ID: 115114. https://doi.org/10.1103/PhysRevB.71.115114

[34] Ziman, J.M. (1974) Prinzipien der Festkorpertheorie. Akademie Verlag, Berlin.

[35] Ziman, J.M. (1967) Electrons and Phonons. The Theory of Transport Phenomena in Solids. Clarendon Press, Oxford.

[36] Smith, C., Janak, J.F. and Adler, B. (1967) Electronic Conduction in Solids. McGraw- 
Hill Book Company, New York.

[37] Wilson, A.H. (1965) The Theory of Metals. Cambridge University Press, Cambridge.

[38] Sonntag, J. (2010) The Effect of the Band Edges on the Seebeck Coefficient. Journal of Physics. Condensed Matter, 22, Article ID: 235501. https://doi.org/10.1088/0953-8984/22/23/235501

[39] Helms, H., Patz, W., Friedemann, C. and Grotzschel, R. (1984) Wiss. Z. Tech. Hochsch. Karl-Marx-Stadt (Chemnitz; Germany), 26, 660.

[40] Zhang, X.X., Liu, H. and Pakhomov, A.B. (2000) Observation of Giant Hall Effect in Non-Magnetic Cermets. Physica B, 279, 81-83. https://doi.org/10.1016/S0921-4526(99)00674-2

[41] Savvides, N., Alister, S.P., Hurd, C.M. and Shiozaki, I. (1982) Localization in the Metallica Regime of Granular $\mathrm{Cu}-\mathrm{SiO}_{2}$ Films. Solid State Communications, 42, 143-145. https://doi.org/10.1016/0038-1098(82)90370-2

[42] Pakhomov, A.B., Yan, X. and Zhao, B. (1995) Giant Hall Effect in Percolating Ferromagnetic Granular Metal-Insulator Films. Applied Physics Letters, 67, 3497-3499. https://doi.org/10.1063/1.115259

[43] Yoshizumi, S., Mael, D., Geballe, T.H. and Greene, R.L. (1985) The Metal-Insulator Transition and Superconductivity in Amorphous Molybdenum-Germanium Alloys. In: Fritzsche, H. and Adler, D., Eds., Localization and Metal-Insulator Transitions, Plenum Press, New York, 77-87. https://doi.org/10.1007/978-1-4613-2517-8_7

[44] Mael, D., Yoshizumi, S. and Geballe, T.H. (1986) Specific Heat of Amorphous $\mathrm{Mo}_{x} \mathrm{Ge}_{1-x}$ through the Metal-Insulator Transition. Physical Review B, 34, 467-470. https://doi.org/10.1103/PhysRevB.34.467

[45] Rogatchev, A.Y., Takeuchi, T. and Mizutani, U. (2000) Comparison of the Specific Heat and the Conductivity of Amorphous $\mathrm{Ti}_{x} \mathrm{Si}_{100-x}$ Alloys across the Metal-Insulator Transition. Physical Review B, 61, 10010-10014. https://doi.org/10.1103/PhysRevB.61.10010

[46] Abkemeier, K.M., Adkins, C.J., Asal, R. and Davis, E.A. (1992) Conductivity and Magnetoresistance of Hydrogenated Amorphous Silicon-Nickel Alloys near the Metal-Insulator Transition. Journal of Physics. Condensed Matter, 4, 9113. https://doi.org/10.1088/0953-8984/4/46/017

[47] Abkemeier, K.M., Adkins, C.J., Asal, R. and Davis, E.A. (1992) Hopping Conduction in Hydrogenated Amorphous $\mathrm{Si}_{1-y} \mathrm{Ni}_{y^{*}}$ Philosophical Magazine B, 65, 675-679. https://doi.org/10.1080/13642819208204902

[48] Schulze, G.E.R. (1967) Metallphysik. Akademie-Verlag, Berlin.

[49] Xiong, P., Xiao, G., Wang, J.Q., Xiao, J.Q., Jiang, J.S. and Chien, C.L. (1992) Extraordinary Hall Effect and Giant Magnetoresistance in the Granular Co-Ag System. Physical Review Letters, 69, 3220-3223. https://doi.org/10.1103/PhysRevLett.69.3220

[50] Masumoto, T. and Maddin, T. (1975) Structural Stability and Mechanical Properties of Amorphous Metals. Materials Science and Engineering, 19, 1-24.

[51] Pakhomov, A.B., Yan, X., Wang, N., Jing, X.N., Zhao, B., Fung, K.K., Xhie, J., Hung, T.F. and Wong, S.K. (1997) On the Origin of the Giant Hall Effect in Magnetic Granular Metals. Physica A, 241, 344-349. https://doi.org/10.1016/S0378-4371(97)00105-2

[52] Wan, C. and Sheng, P. (2002) Quantum Interference and the Giant Hall Effect in Percolating Systems. Physical Review B, 66, Article No. 075309. 
[53] Zhang, X.X., Wan, C., Liu, H., Li, Z.Q., Sheng, P. and Lin, J.J. (2001) Giant Hall Effect in Nonmagnetic Granular Metal Films. Physical Review Letters, 86, 5562-5565. https://doi.org/10.1103/PhysRevLett.86.5562

[54] Liu, H., Zheng, R.K., Wen, G.H. and Zhang, X.X. (2004) Giant Hall Effect in Metal/Insulator Composite Films. Vacuum, 73, 603-610. https://doi.org/10.1016/j.vacuum.2003.12.076

[55] Wen, J.F., Wang, J.F., Zou, W.Q., Zhang, F.M. and Du, Y.W. (2005) Investigation on the Giant Hall Effect of $\left(\mathrm{Fe}_{x} \mathrm{Sn}_{100-x}\right)_{1-y}\left(\mathrm{SiO}_{2}\right)_{y}$ Granular Films. Journal of Alloys and Compounds, 393, 77-80. https://doi.org/10.1016/j.jallcom.2004.10.021

[56] Atkins, P.W. and dePaula, J. (2006) Physikalische Chemie. WILEY-VCH Verlag, Weinheim.

[57] Atkins, P.W. and de Paula, J. (2006) Physical Chemistry. Oxford University Press, Oxford.

[58] Joffe, A.F. (1958) Physik der Halbleiter. Akademie-Verlag, Berlin.

[59] Ricker, T. and Schaumann, G. (1966) Thermoelektrische Eigenschaften reiner Metalle in der Umgebung der Schmelztemperatur. Phys. Kondens. Mater, 5, 31.

[60] Howe, R.A. and Enderby, J.E. (1967) The Thermoelectric Power of Liquid Ag-Au. Philosophical Magazine, 16, 467-476. https://doi.org/10.1080/14786436708220857

[61] Kendall, P.W. (1968) The Absolute Thermoelectric Powers of the Liquid Alkali Metals. Physics and Chemistry of Liquids, 1, 33-48. https://doi.org/10.1080/00319106808083785

[62] Sonntag, J., Ziolkowski, P., Savan, A., Kieschnick, M. and Ludwig, A. (2011) High-Throughput Characterization of the Seebeck Coefficient of a- $\left(\mathrm{Cr}_{1-x} \mathrm{Si}_{x}\right)_{1-y} \mathrm{O}_{y}$ Thin Film Materials Libraries as Verification of the Extended Thermopower Formula. Journal of Physics. Condensed Matter, 23, Article ID: 265501. https://doi.org/10.1088/0953-8984/23/26/265501

[63] Sonntag, J., Ziolkowski, P., Savan, A., Kieschnick, M. and Ludwig, A. (2012) Unpublished.

[64] Ziolkowski, P., Karpinski, G., Platzek, D., Stiewe, C. and Muller, E. (2006) Application Overview of the Potential Seebeck Microscope. 25th International Conference on Thermoelectrics, Vienna, 6-10 August 2006, 684-688.

https://doi.org/10.1109/ICT.2006.331234

[65] Platzek, D., Karpinski, G., Stiewe, C., Ziolkowski, P., Stordeur, M., Engers, B. and Muller, E. (2005) Spatial Resolution of the Seebeck Coefficient Measured on Thermoelectric Thin Films. 3rd European Conference on Thermoelectrics, Nancy, 1-2 September 2005, 119-122. 